\title{
Development of an Improved Frequency Limited Model Order Reduction Technique and Error Bound for Discrete-Time Systems
}

\author{
Sammana BATOOL ${ }^{1}$, Muhammad IMRAN ${ }^{1}$, Dr. Muhammad IMRAN ${ }^{1}$, Ehsan ELAHI ${ }^{2}$, \\ Ayesha MAQBOOL ${ }^{1}$, Syed Amer Ahsan GILANI ${ }^{2}$ \\ ${ }^{1}$ Dept. of Electrical Engineering, Military College of Signals (MCS), National University of Sciences and Technology \\ (NUST), Islamabad, Pakistan \\ 2 Dept. of Information Security (Systems Engineering) MCS, NUST, Islamabad, Pakistan \\ sammana.phdee@students.mcs.edu.pk,imran.phdee@students.mcs.edu.pk,m.imran@mcs.edu.pk, \\ rellahi.msse4mcs@students.mcs.edu.pk, ayesha.maqbool@mcs.edu.pk, gilani.amer@mcs.edu.pk \\ Submitted April 19, 2021 / Accepted July 19, 2021
}

\begin{abstract}
Frequency limited model order reduction algorithm presented by Wang \& Zilouchian for discrete-time systems provide unstable reduced-order models and also do not provide a priori error bound formula. Many stabilitypreserving model order reduction algorithms were presented; however, these methods produce significant approximation errors in the desired frequency interval. An improved algorithm of model order reduction for the discrete-time systems is presented. The proposed technique gives the stable reduced-order model and also provides less approximation error as compared with other algorithms and also provides the formula for the frequency response a priori error bound. Numerical examples provided at the end of the section show the efficacy of the proposed technique.
\end{abstract}

\section{Keywords}

Model order reduction, controllability Gramians, observability Gramians, error bound, balanced truncation

\section{Introduction}

The process of model order reduction (MOR) is to reduce a system from higher-order to its lower order for ease in simulation, analysis, and design of complex systems, filters, controller and circuits, antennas, sensor networks [1-4]. Balanced truncation (BT) [5] is a common and useful scheme to get a stable reduced-order model (ROM) for an original stable system. Moreover, this scheme also has an error bounds formula. However, it uses full frequency interim to get ROM. This encourages the introduction of the frequency weights to perform MOR. Enns [6] extended the work of BT's technique [5] to incorporate frequency weights. Enns [6] method may use single-sided (input/output) and double-sided weights. It gives stable ROM when using only single side weights, whereas, with double-sided weights, stability is not guaranteed. To overcome the problem of Enns [6], many other techniques are given in the literature [7-13].

In some applications, a particular frequency range is of interest. Wang \& Zilouchian (WZ) [14] proposed a limited frequency technique for discrete-time systems without explicit weights. It can yield unstable ROM, and no a priori error bound formula exists.

To overcome the problem of WZ's [14], Ghafoor \& Sreeram (GS) [15] proposed two methods to guarantee the stability of ROM by inducing some variation in input and output related matrices to ensure positive/semi-positive definiteness of some input and output related matrices. Later on, Imran \& Ghafoor (IG) [16] proposed a technique to ensure positive/semi-positive definiteness of some input and output related matrices to get a stable ROM. The work in [15] and [16] guarantees the stability of ROMs and carry a priori error bounds; however, due to extensive variation in input and output related matrices, these methods produce large approximation error and error bound.

In this paper, new measures are proposed to ensure the positive/semi-positive definiteness of the input and the output related matrices by introducing some modifications to the input and output related matrices for discrete-frequency limited Gramians based model reduction. These modifications equally affect the negative eigenvalues, which minimize the variation in these matrices. The stability of ROMs are guaranteed and a priori error bound formula exists. Simulation results show that the proposed method not only ensures the stability of ROMs but also provides better approximation results as compared with other methods in the desired frequency interval, which shows the efficacy of the proposed scheme. 


\begin{tabular}{|c|c|}
\hline Elementary operators & Terminology \\
\hline $\mathbf{G}(z)$ & $\mathbf{C}(z \mathbf{I}-\mathbf{A})^{-1} \mathbf{B}+\mathbf{D} \Leftrightarrow$ state-space realization $\{\mathbf{A}, \mathbf{B}, \mathbf{C}, \mathbf{D}\}$ \\
\hline ROM $\mathbf{G}_{\mathrm{tr}}(z)$ & $\mathbf{C}_{\mathrm{tr}}\left(z \mathbf{I}-\mathbf{A}_{\mathrm{tr}}\right)^{-1} \mathbf{B}_{\mathrm{tr}}+\mathbf{D}_{\mathrm{tr}} \Leftrightarrow$ state-space realization $\left\{\mathbf{A}_{\mathrm{tr}}, \mathbf{B}_{\mathrm{tr}}, \mathbf{C}_{\mathrm{tr}}, \mathbf{D}_{\mathrm{tr}}\right\}$ \\
\hline$\overline{\mathbf{P}}_{\mathrm{c}}>0$ & Positive definite controllability matrix \\
\hline$\overline{\mathbf{P}}_{\mathrm{c}} \geq 0$ & Positive semi-definite controllability matrix \\
\hline$\overline{\mathbf{P}}_{\mathrm{c}}^{\mathrm{T}}$ & Transpose of matrix $\overline{\mathbf{P}}_{\mathrm{c}}^{\mathrm{T}}$ \\
\hline$\overline{\mathbf{P}}_{\mathrm{c}}^{*}$ & Complex conjugate transpose of matrix $\overline{\mathbf{P}}_{\mathrm{c}}^{*}$ \\
\hline$\left|\overline{\mathbf{P}}_{\mathrm{c}}\right|$ & Modulus of matrix $\left|\overline{\mathbf{P}}_{\mathrm{c}}\right|$ \\
\hline $\bar{\lambda}_{i}\left[\overline{\mathbf{P}}_{\mathrm{c}}\right]$ & Eigenvalues of matrix $\left|\overline{\mathbf{P}}_{\mathrm{c}}\right|$ \\
\hline $\bar{\sigma}_{i}\left[\overline{\mathbf{P}}_{\mathrm{c}}\right]$ & Singular-values of matrix $\left|\overline{\mathbf{P}}_{\mathrm{c}}\right|$ \\
\hline$p \mapsto \infty\|x\|_{\infty}:=\max \left|x_{i}\right|$ & Infinity- norm $($ maximum norm $)$ \\
\hline$\sum_{j=1}^{n} \bar{\sigma}_{j}$ & $\bar{\sigma}_{1}+\bar{\sigma}_{2}+\bar{\sigma}_{3}+\ldots+\bar{\sigma}_{n}$ \\
\hline
\end{tabular}

Tab. 1. Elementary operators and terminologies.

\section{Preliminaries}

\subsection{Elementary Operators and Terminologies}

Table 1 briefly summarizes some elementary operators and their terminologies used in this paper.

\subsection{Balancing MOR Techniques}

Consider a discrete-time system be given as:

$$
\begin{aligned}
\mathbf{x}[k+1] & =\mathbf{A} x[k]+\mathbf{B} u[k], \\
\mathbf{y}[k] & =\mathbf{C} x[k]+\mathbf{D} u[k], \\
\mathbf{G}(z) & =\mathbf{C}(z \mathbf{I}-\mathbf{A})^{-1} \mathbf{B}+\mathbf{D}
\end{aligned}
$$

where $\left\{\mathbf{A} \in \mathcal{R}^{n \times n}, \mathbf{B} \in \mathcal{R}^{n \times m}, \mathbf{C} \in \mathcal{R}^{p \times n}, \mathbf{D} \in \mathcal{R}^{p \times m}\right\}$ is its $n^{\text {th }}$ order minimal realization with $m$ number of inputs and $p$ numbers of outputs. The ROM

$$
\begin{aligned}
\mathbf{x}_{\mathrm{tr}}[k+1] & =\mathbf{A}_{\mathrm{tr}} x_{\mathrm{tr}}[k]+\mathbf{B}_{\mathrm{tr}} u[k], \\
\mathbf{y}_{\mathrm{tr}}[k] & =\mathbf{C}_{\mathrm{tr}} x_{\mathrm{tr}}[k]+\mathbf{D}_{\mathrm{tr}} u[k], \\
\mathbf{G}_{\mathrm{tr}}(z) & =\mathbf{C}_{\mathrm{tr}}\left(z \mathbf{I}-\mathbf{A}_{\mathrm{tr}}\right)^{-1} \mathbf{B}_{\mathrm{tr}}+\mathbf{D}_{\mathrm{tr}}
\end{aligned}
$$

is obtained by approximating the actual large-scale system (in the desired frequency interval $\left[\bar{\omega}_{1}, \bar{\omega}_{2}\right]$ ) where $\bar{\omega}_{2}>\bar{\omega}_{1}$, where $\left\{\mathbf{A}_{\mathrm{tr}} \in \mathcal{R}^{r \times r}, \mathbf{B}_{\mathrm{tr}} \in \mathcal{R}^{r \times m}, \mathbf{C}_{\mathrm{tr}} \in \mathcal{R}^{p \times r}, \mathbf{D}_{\mathrm{tr}} \in \mathcal{R}^{p \times m}\right\}$ with $r<<n$. $\overline{\mathbf{P}}_{\mathrm{c}}$ and $\overline{\mathbf{Q}}_{\mathrm{o}}$ are controllability and observability Gramians, respectively

$$
\begin{aligned}
& \overline{\mathbf{P}}_{\mathrm{c}}=\frac{1}{2 \pi} \int_{-\pi}^{\pi}\left(\mathrm{e}^{\mathrm{j} \bar{\omega}} \mathbf{I}-\mathbf{A}\right)^{-1} \mathbf{B} \mathbf{B}^{\mathrm{T}}\left(\mathrm{e}^{-\mathrm{j} \bar{\omega}} \mathbf{I}-\mathbf{A}^{\mathrm{T}}\right)^{-1} \mathrm{~d} \bar{\omega}, \\
& \overline{\mathbf{Q}}_{\mathrm{o}}=\frac{1}{2 \pi} \int_{-\pi}^{\pi}\left(\mathrm{e}^{-\mathrm{j} \bar{\omega}} \mathbf{I}-\mathbf{A}^{\mathrm{T}}\right)^{-1} \mathbf{C}^{\mathrm{T}} \mathbf{C}\left(\mathrm{e}^{\mathrm{j} \bar{\omega}} \mathbf{I}-\mathbf{A}\right)^{-1} \mathrm{~d} \bar{\omega}
\end{aligned}
$$

which are the solution of the following Lyapunov equations:

$$
\begin{aligned}
\mathbf{A} \overline{\mathbf{P}}_{\mathrm{c}} \mathbf{A}^{\mathrm{T}}-\overline{\mathbf{P}}_{\mathrm{c}}+\mathbf{B} \mathbf{B}^{\mathrm{T}}=0, \\
\mathbf{A}^{\mathrm{T}} \overline{\mathbf{Q}}_{\mathrm{o}} \mathbf{A}-\overline{\mathbf{Q}}_{\mathrm{o}}+\mathbf{C}^{\mathrm{T}} \mathbf{C}=0 .
\end{aligned}
$$

\subsubsection{WZ's Technique [14]}

The pioneer discrete-frequency limited approach presented by WZ [14] offers controllability and observabil- ity Gramians at desired discrete-frequency intervals, these Gramians $\overline{\mathbf{P}}_{\mathrm{WZ}}$ and $\overline{\mathbf{Q}}_{\mathrm{WZ}}$ for the discrete-time limited frequency interval, respectively, can be defined as

$$
\begin{aligned}
\overline{\mathbf{P}}_{\mathrm{WZ}} & =\overline{\mathbf{P}}_{\mathrm{c}}\left(\bar{\omega}_{2}\right)-\overline{\mathbf{P}}_{\mathrm{c}}\left(\bar{\omega}_{1}\right) \\
\overline{\mathbf{Q}}_{\mathrm{WZ}} & =\overline{\mathbf{Q}}_{\mathrm{o}}\left(\bar{\omega}_{2}\right)-\overline{\mathbf{Q}}_{\mathrm{o}}\left(\bar{\omega}_{1}\right)
\end{aligned}
$$

where $\overline{\mathbf{P}}_{\mathrm{WZ}}$ and $\overline{\mathbf{Q}}_{\mathrm{WZ}}$ be given as

$$
\begin{aligned}
\overline{\mathbf{P}}_{\mathrm{WZ}} & =\frac{1}{2 \pi} \int_{\delta \bar{\omega}}\left(\mathrm{e}^{\mathrm{j} \bar{\omega}} \mathbf{I}-\mathbf{A}\right)^{-1} \overline{\mathbf{B}}_{\mathrm{WZ}} \overline{\mathbf{B}}_{\mathrm{WZ}}^{\mathrm{T}}\left(\mathrm{e}^{-\mathrm{j} \bar{\omega}} \mathbf{I}-\mathbf{A}^{\mathrm{T}}\right)^{-1} \mathrm{~d} \bar{\omega} \\
\overline{\mathbf{Q}}_{\mathrm{WZ}} & =\frac{1}{2 \pi} \int_{\delta \bar{\omega}}\left(\mathrm{e}^{-\mathrm{j} \bar{\omega}}-\mathbf{A}^{\mathrm{T}}\right)^{-1} \overline{\mathbf{C}}_{\mathrm{WZ}}^{\mathrm{T}} \overline{\mathbf{C}}_{\mathrm{WZ}}\left(\mathrm{e}^{\mathrm{j} \bar{\omega}} \mathbf{I}-\mathbf{A}\right)^{-1} \mathrm{~d} \bar{\omega}
\end{aligned}
$$

where $\delta \bar{\omega}$ is the interval of integration $\left[\bar{\omega}_{1}, \bar{\omega}_{2}\right]$. These Gramians $\overline{\mathbf{P}}_{\mathrm{WZ}}$ and $\overline{\mathbf{Q}}_{\mathrm{WZ}}$ satisfy the following Lyapunov equations

$$
\begin{aligned}
\mathbf{A} \overline{\mathbf{P}}_{\mathrm{WZ}} \mathbf{A}^{\mathrm{T}}-\overline{\mathbf{P}}_{\mathrm{WZ}}+\overline{\mathbf{X}}_{\mathrm{WZ}}=0, \\
\mathbf{A}^{\mathrm{T}} \overline{\mathbf{Q}}_{\mathrm{WZ}} \mathbf{A}-\overline{\mathbf{Q}}_{\mathrm{WZ}}+\overline{\mathbf{Y}}_{\mathrm{WZ}}=0
\end{aligned}
$$

where $\quad \overline{\mathbf{X}}_{\mathrm{WZ}}=\left(\overline{\mathbf{F}}\left(\bar{\omega}_{2}\right)-\overline{\mathbf{F}}\left(\bar{\omega}_{1}\right)\right) \quad \overline{\mathbf{B}}_{\mathrm{WZ}} \overline{\mathbf{B}}_{\mathrm{WZ}}^{\mathrm{T}}+\overline{\mathbf{B}}_{\mathrm{WZ}} \overline{\mathbf{B}}_{\mathrm{WZ}}^{\mathrm{T}}$ $\left(\overline{\mathbf{F}}^{*}\left(\bar{\omega}_{2}\right)-\overline{\mathbf{F}}^{*}\left(\bar{\omega}_{1}\right)\right)$ and $\overline{\mathbf{Y}}_{\mathrm{WZ}}=\left(\overline{\mathbf{F}}\left(\bar{\omega}_{2}\right)-\overline{\mathbf{F}}\left(\bar{\omega}_{1}\right)\right) \overline{\mathbf{C}}_{\mathrm{WZ}}^{\mathrm{T}} \overline{\mathbf{C}}_{\mathrm{WZ}}{ }^{+}$ $\overline{\mathbf{C}}_{\mathrm{WZ}}^{\mathrm{T}} \overline{\mathbf{C}}_{\mathrm{WZ}}\left(\overline{\mathbf{F}}^{*}\left(\bar{\omega}_{2}\right)-\overline{\mathbf{F}}^{*}\left(\bar{\omega}_{1}\right)\right)$ are the input and output related matrices in the limited discrete-frequency intervals, $\overline{\mathbf{F}}(\bar{\omega})=-\frac{\bar{\omega}_{2}-\bar{\omega}_{1}}{4 \pi} \mathbf{I}+\frac{1}{2 \pi} \int_{\underline{\delta} \bar{\omega}}\left(\mathrm{e}^{\mathrm{j} \bar{\omega}} \mathbf{I}-\mathbf{A}\right)^{-1} \mathrm{~d} \bar{\omega}$ and $\overline{\mathbf{F}}^{*}(\bar{\omega})$ is the conjugate transpose of $\overline{\mathbf{F}}(\bar{\omega})$. By eigenvalues decomposition of $\overline{\mathbf{X}}_{\mathrm{WZ}}$ and $\overline{\mathbf{Y}}_{\mathrm{WZ}}$ we have following 


$$
\begin{aligned}
\overline{\mathbf{X}}_{\mathrm{WZ}} & =\overline{\mathbf{U}}_{\mathrm{WZ}}\left[\begin{array}{cc}
\overline{\mathbf{S}}_{\mathrm{WZ}} & 0 \\
0 & \overline{\mathbf{S}}_{\mathrm{WZ}}
\end{array}\right] \overline{\mathbf{U}}_{\mathrm{WZ}}^{\mathrm{T}}, \\
\overline{\mathbf{B}}_{\mathrm{WZ}} & =\overline{\mathbf{U}}_{\mathrm{WZ}}\left[\begin{array}{cc}
\overline{\mathbf{S}}_{\mathrm{WZ}_{1}}^{1 / 2} & 0 \\
0 & \overline{\mathbf{S}}_{\mathrm{WZ}_{2}}^{1 / 2}
\end{array}\right]=\overline{\mathbf{U}}_{\mathrm{WZ}} \overline{\mathbf{S}}_{\mathrm{WZ}}^{1 / 2}, \\
\overline{\mathbf{Y}}_{\mathrm{WZ}} & =\overline{\mathbf{V}}_{\mathrm{WZ}}\left[\begin{array}{cc}
\overline{\mathbf{R}}_{\mathrm{WZ}_{1}} & 0 \\
0 & \overline{\mathbf{R}}_{\mathrm{WZ}}
\end{array}\right] \overline{\mathbf{V}}_{\mathrm{WZ}}^{\mathrm{T}}, \\
\overline{\mathbf{C}}_{\mathrm{WZ}} & =\left[\begin{array}{cc}
\overline{\mathbf{R}}_{\mathrm{WZ}}^{1 / 2} & 0 \\
0 & \overline{\mathbf{R}}_{\mathrm{WZ}}^{1 / 2}
\end{array}\right] \overline{\mathbf{V}}_{\mathrm{WZ}}^{\mathrm{T}}=\overline{\mathbf{R}}_{\mathrm{WZ}}^{1 / 2} \overline{\mathbf{V}}_{\mathrm{WZ}}^{\mathrm{T}}
\end{aligned}
$$

where

$$
\begin{aligned}
& \overline{\mathbf{S}}_{\mathrm{WZ}_{1}}=\left[\begin{array}{cccc}
\bar{s}_{1} & 0 & \cdots & 0 \\
0 & \bar{s}_{2} & \cdots & 0 \\
0 & 0 & \cdots & \bar{s}_{q-1}
\end{array}\right], \overline{\mathbf{S}}_{\mathrm{WZ}_{2}}=\left[\begin{array}{cccc}
\bar{s}_{q} & 0 & \cdots & 0 \\
0 & \bar{s}_{q+1} & \cdots & 0 \\
0 & 0 & \cdots & \bar{s}_{n}
\end{array}\right], \\
& \overline{\mathbf{R}}_{\mathrm{WZ}_{1}}=\left[\begin{array}{cccc}
\bar{r}_{1} & 0 & \cdots & 0 \\
0 & \bar{r}_{2} & \cdots & 0 \\
0 & 0 & \cdots & \bar{r}_{k-1}
\end{array}\right], \overline{\mathbf{R}}_{\mathrm{WZ}_{2}}=\left[\begin{array}{cccc}
\bar{r}_{k} & 0 & \cdots & 0 \\
0 & \bar{r}_{k+1} & \cdots & 0 \\
0 & 0 & \cdots & \bar{r}_{n}
\end{array}\right]
\end{aligned}
$$

$\overline{\mathbf{X}}_{\mathrm{WZ}}$ and $\overline{\mathbf{Y}}_{\mathrm{WZ}}$ contain $(q-1)$ and $(k-1)$ number of positive eigenvalues, respectively. Let a transformation matrix $\overline{\mathbf{T}}_{\mathrm{WZ}}$ is obtained as:

$$
\overline{\mathbf{T}}_{\mathrm{WZ}}^{\mathrm{T}} \overline{\mathbf{Q}}_{\mathrm{WZ}} \overline{\mathbf{T}}_{\mathrm{WZ}}=\overline{\mathbf{T}}_{\mathrm{WZ}}^{-1} \overline{\mathbf{P}}_{\mathrm{WZ}} \overline{\mathbf{T}}_{\mathrm{WZ}}^{-\mathrm{T}}=\operatorname{diag}\left\{\bar{\sigma}_{1}, \bar{\sigma}_{2}, \ldots, \bar{\sigma}_{n}\right\}
$$

and the ROM G $\mathbf{G}_{\mathrm{tr}}(z)=\overline{\mathbf{C}}_{\mathrm{tr}}\left(z \mathbf{I}-\overline{\mathbf{A}}_{\mathrm{tr}}\right)^{-1} \overline{\mathbf{B}}_{\mathrm{tr}}+\overline{\mathbf{D}}_{\mathrm{tr}}$ obtained is

$$
\begin{aligned}
\overline{\mathbf{T}}_{\mathrm{WZ}}^{-1} \mathbf{A} \overline{\mathbf{T}}_{\mathrm{WZ}} & =\overline{\mathbf{A}}=\left[\begin{array}{ll}
\overline{\mathbf{A}}_{\mathrm{tr}} & \overline{\mathbf{A}}_{12} \\
\overline{\mathbf{A}}_{21} & \overline{\mathbf{A}}_{22}
\end{array}\right], \overline{\mathbf{T}}_{\mathrm{WZ}}^{-1} \mathbf{B}=\overline{\mathbf{B}}=\left[\begin{array}{c}
\overline{\mathbf{B}}_{\mathrm{tr}} \\
\overline{\mathbf{B}}_{2}
\end{array}\right] \\
\mathbf{C} \overline{\mathbf{T}}_{\mathrm{WZ}} & =\overline{\mathbf{C}}=\left[\begin{array}{ll}
\mathbf{C}_{\mathrm{tr}} & \overline{\mathbf{C}}_{2}
\end{array}\right], \quad \mathbf{D}=\overline{\mathbf{D}}_{\mathrm{tr}}
\end{aligned}
$$

where $\bar{\sigma}_{j} \geq \bar{\sigma}_{j+1}, j=1,2,3, \ldots, n-1, \bar{\sigma}_{r}>\bar{\sigma}_{r+1}$ where $r$ is the order of the ROM and $\overline{\mathbf{T}}_{\mathrm{WZ}}$ is a similarity transformation matrix used to obtain the transformed system. The ROM can be acquired by truncating the transformed system.

Remark 1 For the discrete frequency-range $\left[\bar{\omega}_{1}, \bar{\omega}_{2}\right]=$ $[-\pi, \pi], \quad \lim _{\bar{\omega}_{\mapsto[-\pi, \pi]}} \overline{\mathbf{P}}_{\mathrm{c}}[\bar{\omega}]=\overline{\mathbf{P}}_{\mathrm{c}}=\overline{\mathbf{P}}_{\mathrm{WZ}}$, $\lim _{\bar{\omega}_{\mapsto[-\pi, \pi]}} \overline{\mathbf{Q}}_{\mathrm{o}}[\bar{\omega}]=\overline{\mathbf{Q}}_{\mathrm{o}}=\overline{\mathbf{Q}}_{\mathrm{WZ}}$, where $\overline{\mathbf{P}}_{\mathrm{c}}[\bar{\omega}]$ and $\overline{\mathbf{Q}}_{\mathrm{o}}[\bar{\omega}]$ are obtained using Parseval's relationship [17], the ROMs obtained using the WZ [14] and the BT [5] are same.

Remark 2 WZ [14] failed to make sure the stability of ROMs because input/output associated matrices $\overline{\mathbf{X}}_{\mathrm{WZ}}$ and $\overline{\mathbf{Y}}_{\mathrm{WZ}}$ may no longer be positive definite or semi-definite [15].

\subsubsection{Existing Stability Preserving Frequency Limited Techniques}

To overcome the main drawback of WZ [14], GS [15], and IG [16] proposed stability preserving MOR approaches. However, these techniques produce large approximation error and extensive a priori error bound formula due to large variation in input and output related matrices. Let the controllability $\overline{\mathbf{P}}_{\mathrm{E}}$ and observability $\overline{\mathbf{Q}}_{\mathrm{E}}$ Gramians, respectively, satisfy

$$
\begin{array}{r}
\mathbf{A} \overline{\mathbf{P}}_{\mathrm{E}} \mathbf{A}^{\mathrm{T}}-\overline{\mathbf{P}}_{\mathrm{E}}+\overline{\mathbf{B}}_{\mathrm{E}} \overline{\mathbf{B}}_{\mathrm{E}}^{\mathrm{T}}=0, \\
\mathbf{A}^{\mathrm{T}} \overline{\mathbf{Q}}_{\mathrm{E}} \mathbf{A}-\overline{\mathbf{Q}}_{\mathrm{E}}+\overline{\mathbf{C}}_{\mathrm{E}}^{\mathrm{T}} \overline{\mathbf{C}}_{\mathrm{E}}=0
\end{array}
$$

where $\overline{\mathbf{B}}_{\mathrm{E}} \in\left\{\overline{\mathbf{B}}_{\mathrm{E}_{1}}[15], \overline{\mathbf{B}}_{\mathrm{E}_{2}}\right.$ [15], $\overline{\mathbf{B}}_{\mathrm{E}_{3}}$ [16] $\}$ and $\overline{\mathbf{C}}_{\mathrm{E}} \in$ $\left\{\overline{\mathbf{C}}_{\mathrm{E}_{1}}[15], \overline{\mathbf{C}}_{\mathrm{E}_{2}}[15], \overline{\mathbf{C}}_{\mathrm{E}_{3}}[16]\right\}$.

$$
\begin{aligned}
& \overline{\mathbf{B}}_{\mathrm{E}_{1}}=\overline{\mathbf{U}}_{\mathrm{E}_{1}}\left[\begin{array}{cc}
\overline{\mathbf{S}}_{\mathrm{WZ}_{1}}^{1 / 2} & 0 \\
0 & \left|\overline{\mathbf{S}}_{\mathrm{WZ}_{2}}\right|^{1 / 2}
\end{array}\right], \\
& \overline{\mathbf{B}}_{\mathrm{E}_{2}}=\overline{\mathbf{U}}_{\mathrm{E}_{2}}\left[\begin{array}{cc}
\overline{\mathbf{S}}_{\mathrm{WZ}_{1}}^{1 / 2} & 0 \\
0 & 0
\end{array}\right] \text {, } \\
& \overline{\mathbf{B}}_{\mathrm{E}_{3}}= \begin{cases}\overline{\mathbf{U}}_{\mathrm{E}_{3}}\left(\overline{\mathbf{S}}_{\mathrm{WZ}}-\bar{s}_{n} I\right)^{1 / 2} & \text { for } \bar{s}_{n}<0, \\
\overline{\mathbf{U}}_{\mathrm{E}_{3}} \overline{\mathbf{S}}_{\mathrm{WZ}}^{1 / 2} & \text { for } \bar{s}_{n} \geq 0,\end{cases} \\
& \overline{\mathbf{C}}_{\mathrm{E}_{1}}=\left[\begin{array}{cc}
\overline{\mathbf{R}}_{\mathrm{WZ}}^{1 / 2} & 0 \\
0 & \left|\overline{\mathbf{R}}_{\mathrm{WZ}_{2}}\right|^{1 / 2}
\end{array}\right] \overline{\mathbf{V}}_{\mathrm{E}_{1}}^{\mathrm{T}}, \\
& \overline{\mathbf{C}}_{\mathrm{E}_{2}}=\left[\begin{array}{cc}
\overline{\mathbf{R}}_{\mathrm{WZ}}^{1 / 2} & 0 \\
0 & 0
\end{array}\right] \overline{\mathbf{V}}_{\mathrm{E}_{2}}^{\mathrm{T}}, \\
& \overline{\mathbf{C}}_{\mathrm{E}_{3}}= \begin{cases}\left(\overline{\mathbf{R}}_{\mathrm{WZ}}-\bar{r}_{n} \mathbf{I}\right)^{1 / 2} \overline{\mathbf{V}}_{\mathrm{E}_{3}}^{\mathrm{T}} & \text { for } \bar{r}_{n}<0, \\
\overline{\mathbf{R}}_{\mathrm{WZ}}^{1 / 2} \overline{\mathbf{V}}_{\mathrm{E}_{3}}^{\mathrm{T}} & \text { for } \bar{r}_{n} \geq 0 .\end{cases}
\end{aligned}
$$

Let a transformation matrix $\overline{\mathbf{T}}_{\mathrm{E}}$ is obtained as:

$$
\overline{\mathbf{T}}_{\mathrm{E}}^{\mathrm{T}} \overline{\mathbf{Q}}_{\mathrm{E}} \overline{\mathbf{T}}_{\mathrm{E}}=\overline{\mathbf{T}}_{\mathrm{E}}^{-1} \overline{\mathbf{P}}_{\mathrm{E}} \overline{\mathbf{T}}_{\mathrm{E}}^{-\mathrm{T}}=\left[\begin{array}{cccc}
\bar{\sigma}_{1} & 0 & \cdots & 0 \\
0 & \bar{\sigma}_{2} & \cdots & 0 \\
\cdots & \cdots & \ddots & \cdots \\
0 & 0 & \cdots & \bar{\sigma}_{n}
\end{array}\right]
$$

and the ROM G $\mathbf{G}_{\text {tr }}(z)=\overline{\mathbf{C}}_{\text {tr }}\left(z \mathbf{I}-\overline{\mathbf{A}}_{\text {tr }}\right)^{-1} \overline{\mathbf{B}}_{\text {tr }}+\overline{\mathbf{D}}_{\text {tr }}$ obtained is

$$
\begin{aligned}
\overline{\mathbf{T}}_{\mathrm{E}}^{-1} \mathbf{A} \overline{\mathbf{T}}_{\mathrm{E}} & =\overline{\mathbf{A}}=\left[\begin{array}{ll}
\overline{\mathbf{A}}_{\mathrm{tr}} & \overline{\mathbf{A}}_{12} \\
\overline{\mathbf{A}}_{21} & \overline{\mathbf{A}}_{22}
\end{array}\right], \overline{\mathbf{T}}_{\mathrm{E}}^{-1} \mathbf{B}=\overline{\mathbf{B}}=\left[\begin{array}{c}
\overline{\mathbf{B}}_{\mathrm{tr}} \\
\overline{\mathbf{B}}_{2}
\end{array}\right] \\
\mathbf{C} \overline{\mathbf{T}}_{\mathrm{E}} & =\overline{\mathbf{C}}=\left[\begin{array}{ll}
\mathbf{C}_{\mathrm{tr}} & \overline{\mathbf{C}}_{2}
\end{array}\right], \quad \mathbf{D}=\overline{\mathbf{D}}_{\mathrm{tr}}
\end{aligned}
$$

where $\bar{\sigma}_{j} \geq \bar{\sigma}_{j+1}, j=1,2,3, \ldots, n-1, \bar{\sigma}_{r}>\bar{\sigma}_{r+1}$ where $r$ is the order of the ROM and $\overline{\mathbf{T}}_{\mathrm{E}}$ is a similarity transformation matrix used to obtain the transformed system. The ROM can be acquired by truncating the transformed system.

Remark 3 Since $\overline{\mathbf{X}}_{\mathrm{WZ}} \leq \overline{\mathbf{B}}_{\mathrm{E}} \overline{\mathbf{B}}_{\mathrm{E}}^{\mathrm{T}} \geq 0, \overline{\mathbf{Y}}_{\mathrm{WZ}} \leq \overline{\mathbf{C}}_{\mathrm{E}}^{\mathrm{T}} \overline{\mathbf{C}}_{\mathrm{E}} \geq 0$, $\overline{\mathbf{P}}_{\mathrm{E}}>0$ and $\overline{\mathbf{Q}}_{\mathrm{E}}>0$. Therefore, the realization $\left(\mathbf{A}, \overline{\mathbf{B}}_{\mathrm{E}}, \overline{\mathbf{C}}_{\mathrm{E}}\right)$ is minimal and the stability of the ROM is guaranteed.

Remark 4 Since for each input related matrix $\overline{\mathbf{B}}_{\mathrm{E}} \in$ $\left\{\overline{\mathbf{B}}_{\mathrm{E}_{1}}\right.$ [15], $\overline{\mathbf{B}}_{\mathrm{E}_{2}}$ [15], $\overline{\mathbf{B}}_{\mathrm{E}_{3}}$ [16] $\}$ and for each output related matrix $\overline{\mathbf{B}}_{C} \in\left\{\overline{\mathbf{C}}_{\mathrm{E}_{1}}\right.$ [15], $\overline{\mathbf{C}}_{\mathrm{E}_{2}}$ [15], $\overline{\mathbf{C}}_{\mathrm{E}_{3}}$ [16] $\}$ ensure positive/semi-positive definiteness of input and output related matrices, which results positive/semi-positive definiteness of $\overline{\mathbf{P}}_{\mathrm{E}} \in\left\{\overline{\mathbf{P}}_{\mathrm{E}_{1}}[15], \overline{\mathbf{P}}_{\mathrm{E}_{2}}[15], \overline{\mathbf{P}}_{\mathrm{E}_{3}}[16]\right\}$ and $\overline{\mathbf{Q}}_{\mathrm{E}} \in\left\{\overline{\mathbf{Q}}_{\mathrm{E}_{1}}[15]\right.$, $\overline{\mathbf{Q}}_{\mathrm{E}_{2}}$ [15], $\overline{\mathbf{Q}}_{\mathrm{E}_{3}}$ [16]\} in a unique way. This leads to different transformation matrix $\overline{\mathbf{T}}_{\mathrm{E}} \in\left\{\overline{\mathbf{T}}_{\mathrm{E}_{1}}[15], \overline{\mathbf{T}}_{\mathrm{E}_{2}}\right.$ [15], $\left.\overline{\mathbf{T}}_{\mathrm{E}_{3}}[16]\right\}$, which subsequently results in three existing stability preserving model order reduction techniques. 


\section{Main Results}

The existing stability preserving techniques GS [15] and IG [16] introduced some modification in the input and the output related matrices $\overline{\mathbf{X}}_{\mathrm{WZ}}$ and $\overline{\mathbf{Y}}_{\mathrm{WZ}}$ respectively to ensure positive/semi-positive definiteness. In 1-st algorithm of GS [15] modifications are done by taking absolute of eigenvalues of the input and the output related matrices; consequently, positive/semi-positive definiteness of the input and the output related matrices which ensure the stability of ROMs; however, by taking the absolute it may cause an unequal effect on eigenvalues which results in a large variation in some eigenvalues and small variation in some eigenvalues which leads to producing a large approximation error. In 2-nd algorithm of GS [15] stability is ensured by truncating negative eigenvalues, which result in loss of information of negative eigenvalues that leads to a large approximation error, whereas, in IG [16] the stability is ensured by subtracting all the eigenvalues with least eigenvalues which made the last eigenvalue zero that leads to large approximation error. Keeping the above in view, all methods produce large variations in input/output related matrices, which may affect the input/output properties of the original system.

In this paper, new measures are proposed for the input/output related matrices by introducing the concept of the norm of the negative part of the eigenvalues of input/output related matrices without affecting the positive part of the eigenvalues. These modifications equally affect these negative eigenvalues which minimize the variation in the input and the output related matrices, consequently, ensure the positive/semi-positive definiteness the of input and the output related matrices that yield a stable ROM and produces low-frequency response error along with low error bound with minimum variation.

Let the new virtual/fictitious controllability $\overline{\mathbf{P}}_{\mathrm{SB}}$ and observability $\overline{\mathbf{Q}}_{\mathrm{SB}}$ Gramians are computed as

$$
\begin{array}{r}
\mathbf{A} \overline{\mathbf{P}}_{\mathrm{SB}} \mathbf{A}^{\mathrm{T}}-\overline{\mathbf{P}}_{\mathrm{SB}}+\overline{\mathbf{X}}_{\mathrm{SB}}=0, \\
\mathbf{A}^{\mathrm{T}} \overline{\mathbf{Q}}_{\mathrm{SB}} \mathbf{A}-\overline{\mathbf{Q}}_{\mathrm{SB}}+\overline{\mathbf{Y}}_{\mathrm{SB}}=0 .
\end{array}
$$

where $\overline{\mathbf{X}}_{\mathrm{SB}}=\overline{\mathbf{B}}_{\mathrm{SB}} \overline{\mathbf{B}}_{\mathrm{SB}}^{\mathrm{T}}$ and $\overline{\mathbf{Y}}_{\mathrm{SB}}=\overline{\mathbf{C}}_{\mathrm{SB}}^{\mathrm{T}} \overline{\mathbf{C}}_{\mathrm{SB}}$. By eigenvalues decomposition of $\overline{\mathbf{X}}_{\mathrm{SB}}$ and $\overline{\mathbf{Y}}_{\mathrm{SB}}$ we have

$$
\begin{aligned}
\overline{\mathbf{X}}_{\mathrm{SB}} & =\overline{\mathbf{U}}_{\mathrm{SB}} \overline{\mathbf{S}}_{\mathrm{SB}} \overline{\mathbf{U}}_{\mathrm{SB}}^{\mathrm{T}}, \\
\overline{\mathbf{Y}}_{\mathrm{SB}} & =\overline{\mathbf{V}}_{\mathrm{SB}} \overline{\mathbf{R}}_{\mathrm{SB}} \overline{\mathbf{V}}_{\mathrm{SB}}^{\mathrm{T}} .
\end{aligned}
$$

The new virtual/fictitious input and output related matrices respectively are given as $\overline{\mathbf{B}}_{\mathrm{SB}}$ and $\overline{\mathbf{C}}_{\mathrm{SB}}$ where

$$
\begin{aligned}
& \overline{\mathbf{B}}_{\mathrm{SB}}=\overline{\mathbf{U}}_{\mathrm{SB}}\left[\begin{array}{cc}
\overline{\mathbf{S}}_{\mathrm{SB}_{1}}^{1 / 2} & 0 \\
0 & \overline{\mathbf{S}}_{\mathrm{SB}_{2}}^{1 / 2}
\end{array}\right]=\overline{\mathbf{U}}_{\mathrm{SB}} \overline{\mathbf{S}}_{\mathrm{SB}}^{1 / 2}, \\
& \overline{\mathbf{C}}_{\mathrm{SB}}=\left[\begin{array}{cc}
\overline{\mathbf{R}}_{\mathrm{SB}_{1}}^{1 / 2} & 0 \\
0 & \overline{\mathbf{R}}_{\mathrm{SB}_{2}}^{1 / 2}
\end{array}\right] \overline{\mathbf{V}}_{\mathrm{SB}}^{\mathrm{T}}=\overline{\mathbf{R}}_{\mathrm{SB}}^{1 / 2} \overline{\mathbf{V}}_{\mathrm{SB}}^{\mathrm{T}}
\end{aligned}
$$

where $\overline{\mathbf{S}}_{\mathrm{SB}_{1}}=\overline{\mathbf{S}}_{\mathrm{WZ}_{1}}, \overline{\mathbf{R}}_{\mathrm{SB}_{1}}=\overline{\mathbf{R}}_{\mathrm{WZ}_{1}}$,

$$
\begin{aligned}
\overline{\mathbf{S}}_{\mathrm{SB}_{2}} & =\left[\begin{array}{cccc}
\hat{s}_{q} & 0 & \cdots & 0 \\
0 & \hat{s}_{q+1} & \cdots & 0 \\
0 & 0 & \cdots & \hat{s}_{n}
\end{array}\right], \overline{\mathbf{R}}_{\mathrm{SB}_{2}}=\left[\begin{array}{cccc}
\hat{r}_{k} & 0 & \cdots & 0 \\
0 & \hat{r}_{k+1} & \cdots & 0 \\
0 & 0 & \cdots & \hat{r}_{n}
\end{array}\right], \\
\hat{s}_{q+l} & =\left\|\left(\bar{s}_{q+l}, \hat{s}\right)\right\|_{(n-q)}=\left(\left|\bar{s}_{q+l}\right|^{(n-q)}+|\hat{s}|^{(n-q)}\right)^{1 /(n-q)}, \\
\hat{s}_{n} & =\left\|\left(\bar{s}_{n}, \hat{s}\right)\right\|_{(n-q)}=\left(\left|\bar{s}_{n}\right|^{(n-q)}+|\hat{s}|^{(n-q)}\right)^{1 /(n-q)}, \\
\hat{r}_{k+h} & =\left\|\left(\bar{r}_{k+h}, \hat{r}\right)\right\|_{(n-k)}=\left(\left|\bar{r}_{k+h}\right|^{(n-k)}+|\hat{r}|^{(n-k)}\right)^{1 /(n-k)}, \\
\hat{r}_{n} & =\left\|\left(\bar{r}_{n}, \hat{r}\right)\right\|_{(n-k)}=\left(\left|\bar{r}_{n}\right|^{(n-k)}+|\hat{r}|^{(n-k)}\right)^{1 /(n-k)}
\end{aligned}
$$

for $l=0,1, \ldots, n-q-1$, and for $h=0,1, \ldots, n-k-1 . \overline{\mathbf{S}}_{\mathrm{SB}_{2}}$ and $\overline{\mathbf{R}}_{\mathrm{SB}_{2}}$ contain $(n-q)$ and $(n-k)$ number of modified positive eigenvalues, respectively, where $\hat{s}=\sum_{i=q}^{n} \bar{s}_{i}$ and $\hat{r}=\sum_{i=k}^{n} \bar{r}_{i}$. Let the similarity transformation matrix $\overline{\mathbf{T}}_{\mathrm{SB}}$ is calculated as

$$
\overline{\mathbf{T}}_{\mathrm{SB}}^{\mathrm{T}} \overline{\mathbf{Q}}_{\mathrm{SB}} \overline{\mathbf{T}}_{\mathrm{SB}}=\overline{\mathbf{T}}_{\mathrm{SB}}^{-1} \overline{\mathbf{P}}_{\mathrm{SB}} \overline{\mathbf{T}}_{\mathrm{SB}}^{-\mathrm{T}}=\operatorname{diag}\left\{\bar{\sigma}_{1}, \bar{\sigma}_{2}, \bar{\sigma}_{3} \ldots, \bar{\sigma}_{n}\right\}
$$

where $\bar{\sigma}_{j} \geq \bar{\sigma}_{j+1}$ and $\bar{\sigma}_{r} \geq \bar{\sigma}_{r+1}$. The ROM $\mathbf{G}_{\mathrm{tr}}(z)=$ $\hat{\mathbf{C}}_{\mathrm{tr}}\left(z \mathbf{I}-\hat{\mathbf{A}}_{\mathrm{tr}}\right)^{-1} \hat{\mathbf{B}}_{\mathrm{tr}}+\hat{\mathbf{D}}_{\mathrm{tr}}$ is obtained as

$$
\begin{aligned}
\overline{\mathbf{T}}_{\mathrm{SB}}^{-1} \mathbf{A} \overline{\mathbf{T}}_{\mathrm{SB}} & =\hat{\mathbf{A}}=\left[\begin{array}{ll}
\hat{\mathbf{A}}_{\mathrm{tr}} & \hat{\mathbf{A}}_{12} \\
\hat{\mathbf{A}}_{21} & \hat{\mathbf{A}}_{22}
\end{array}\right], \overline{\mathbf{T}}_{\mathrm{SB}}^{-1} \mathbf{B}=\hat{\mathbf{B}}=\left[\begin{array}{c}
\hat{\mathbf{B}}_{\mathrm{tr}} \\
\hat{\mathbf{B}}_{2},
\end{array}\right](26) \\
\mathbf{C} \overline{\mathbf{T}}_{\mathrm{SB}} & =\hat{\mathbf{C}}=\left[\begin{array}{ll}
\hat{\mathbf{C}}_{\mathrm{tr}} & \hat{\mathbf{C}}_{2}
\end{array}\right], \quad \mathbf{D}=\hat{\mathbf{D}}_{\mathrm{tr}} .
\end{aligned}
$$

Remark 5 Since $\overline{\mathbf{X}}_{\mathrm{WZ}} \leq \overline{\mathbf{B}}_{\mathrm{SB}} \overline{\mathbf{B}}_{\mathrm{SB}}^{\mathrm{T}} \geq 0, \overline{\mathbf{Y}}_{\mathrm{WZ}} \leq \overline{\mathbf{C}}_{\mathrm{SB}}^{\mathrm{T}} \overline{\mathbf{C}}_{\mathrm{SB}} \geq$ $0, \overline{\mathbf{P}}_{\mathrm{SB}}>0$ and $\overline{\mathbf{Q}}_{\mathrm{SB}}>0$. Therefore, the realization $\left(\mathbf{A}, \overline{\mathbf{B}}_{\mathrm{SB}}, \overline{\mathbf{C}}_{\mathrm{SB}}\right)$ is minimal and the stability of the ROM is guaranteed.

Theorem 1 Let $\operatorname{rank}\left[\overline{\mathbf{B}}_{\mathrm{SB}} \mathbf{B}\right]=\operatorname{rank}\left[\overline{\mathbf{B}}_{\mathrm{SB}}\right]$ and $\operatorname{rank}\left[\begin{array}{c}\overline{\mathbf{C}}_{\mathrm{SB}} \\ \mathbf{C}\end{array}\right]=\operatorname{rank}\left[\overline{\mathbf{C}}_{\mathrm{SB}}\right]$ following error bound holds

$$
\left\|\mathbf{G}(z)-\mathbf{G}_{\mathrm{tr}}(z)\right\|_{\infty} \leq 2\left\|\overline{\mathbf{L}}_{\mathrm{SB}}\right\|\left\|\overline{\mathbf{K}}_{\mathrm{SB}}\right\| \sum_{j=r+1}^{n} \bar{\sigma}_{j}
$$

where

$$
\begin{aligned}
\overline{\mathbf{L}}_{\mathrm{SB}} & = \begin{cases}\mathbf{C} \overline{\mathbf{V}}_{\mathrm{SB}} \overline{\mathbf{R}}_{\mathrm{SB}}^{-1 / 2} & \text { if } \overline{\mathbf{R}}_{\mathrm{WZ}} \text { exists, } \\
\mathbf{C} \overline{\mathbf{V}}_{\mathrm{WZ}} \overline{\mathbf{R}}_{\mathrm{WZ}}^{-1 / 2} & \text { otherwise, }\end{cases} \\
\overline{\mathbf{K}}_{\mathrm{SB}} & = \begin{cases}\overline{\mathbf{S}}_{\mathrm{SB}}^{-1 / 2} \overline{\mathbf{U}}_{\mathrm{SB}}^{\mathrm{T}} \mathbf{B} & \text { if } \overline{\mathbf{S}}_{\mathrm{WZ}} \text { exists, } \\
\overline{\mathbf{S}}_{\mathrm{WZ}}^{-1 / 2} \overline{\mathbf{U}}_{\mathrm{WZ}}^{\mathrm{T}} \mathbf{B} & \text { otherwise }\end{cases}
\end{aligned}
$$

Proof: The proof follow similar to [16]; however, it is presented here for completeness. Since $\operatorname{rank}\left[\overline{\mathbf{B}}_{\mathrm{SB}} \mathbf{B}\right]=$ $\operatorname{rank}\left[\overline{\mathbf{B}}_{\mathrm{SB}}\right]$ and rank $\left[\begin{array}{c}\overline{\mathbf{C}}_{\mathrm{SB}} \\ \mathbf{C}\end{array}\right]=\operatorname{rank}\left[\overline{\mathbf{C}}_{\mathrm{SB}}\right]$, the relationships $\mathbf{B}=\overline{\mathbf{B}}_{\mathrm{SB}} \overline{\mathbf{K}}_{\mathrm{SB}}$ and $\mathbf{C}=\overline{\mathbf{L}}_{\mathrm{SB}} \overline{\mathbf{C}}_{\mathrm{SB}}$ hold. By partition$\operatorname{ing} \overline{\mathbf{B}}_{\mathrm{SB}}=\left[\begin{array}{c}\overline{\mathbf{B}}_{\mathrm{SB}_{1}} \\ \overline{\mathbf{B}}_{\mathrm{SB}_{2}}\end{array}\right], \overline{\mathbf{C}}_{\mathrm{SB}}=\left[\begin{array}{cc}\overline{\mathbf{C}}_{\mathrm{SB}_{1}} & \overline{\mathbf{C}}_{\mathrm{SB}_{2}}\end{array}\right]$ and substituting 
$\hat{\mathbf{B}}_{\mathrm{tr}}=\overline{\mathbf{B}}_{\mathrm{SB}_{1}} \overline{\mathbf{K}}_{\mathrm{SB}}, \hat{\mathbf{C}}_{\mathrm{tr}}=\overline{\mathbf{L}}_{\mathrm{SB}} \overline{\mathbf{C}}_{\mathrm{SB}_{1}}$, respectively, yields

$$
\begin{aligned}
\left\|\mathbf{G}(z)-\mathbf{G}_{\mathrm{tr}}(z)\right\|_{\infty}= & \left\|\mathbf{C}(z \mathbf{I}-\mathbf{A})^{-1} \mathbf{B}-\mathbf{C}_{\mathrm{tr}}\left(z \mathbf{I}-\hat{\mathbf{A}}_{\mathrm{tr}}\right)^{-1} \hat{\mathbf{B}}_{\mathrm{tr}}\right\|_{\infty} \\
= & \| \overline{\mathbf{L}}_{\mathrm{SB}} \overline{\mathbf{C}}_{\mathrm{SB}}(z \mathbf{I}-\mathbf{A})^{-1} \overline{\mathbf{B}}_{\mathrm{SB}} \overline{\mathbf{K}}_{\mathrm{SB}} \\
& -\overline{\mathbf{L}}_{\mathrm{SB}} \overline{\mathbf{C}}_{\mathrm{SB}_{1}}\left(z \mathbf{I}-\hat{\mathbf{A}}_{\mathrm{tr}}\right)^{-1} \overline{\mathbf{B}}_{\mathrm{SB}} \overline{\mathbf{K}}_{\mathrm{SB}} \|_{\infty} \\
= & \| \overline{\mathbf{L}}_{\mathrm{SB}}\left(\overline{\mathbf{C}}_{\mathrm{SB}}(z \mathbf{I}-\mathbf{A})^{-1} \overline{\mathbf{B}}_{\mathrm{SB}}\right. \\
& \left.-\overline{\mathbf{C}}_{\mathrm{SB}_{1}}\left(z \mathbf{I}-\hat{\mathbf{A}}_{\mathrm{tr}}\right)^{-1} \overline{\mathbf{B}}_{\mathrm{SB}_{1}}\right) \overline{\mathbf{K}}_{\mathrm{SB}} \|_{\infty} \\
\leq & \left\|\overline{\mathbf{L}}_{\mathrm{SB}}\right\| \|\left(\overline{\mathbf{C}}_{\mathrm{SB}}(z \mathbf{I}-\mathbf{A})^{-1} \overline{\mathbf{B}}_{\mathrm{SB}}\right. \\
& \left.-\overline{\mathbf{C}}_{\mathrm{SB}_{1}}\left(z \mathbf{I}-\hat{\mathbf{A}}_{\mathrm{tr}}\right)^{-1} \overline{\mathbf{B}}_{\mathrm{SB}_{1}}\right)\left\|_{\infty}\right\| \overline{\mathbf{K}}_{\mathrm{SB}} \| .
\end{aligned}
$$

If $\left\{\hat{\mathbf{A}}_{\mathrm{tr}}, \overline{\mathbf{B}}_{\mathrm{SB}_{1}}, \overline{\mathbf{C}}_{\mathrm{SB}_{1}}\right\}$ is the model obtained after reduction of the original system $\left\{\mathbf{A}, \overline{\mathbf{B}}_{\mathrm{SB}}, \overline{\mathbf{C}}_{\mathrm{SB}}\right\}$.

$\left\|\left(\overline{\mathbf{C}}_{\mathrm{SB}}(z \mathbf{I}-\mathbf{A})^{-1} \overline{\mathbf{B}}_{\mathrm{SB}}-\overline{\mathbf{C}}_{\mathrm{SB}_{1}}\left(z \mathbf{I}-\hat{\mathbf{A}}_{\mathrm{tr}}\right)^{-1} \overline{\mathbf{B}}_{\mathrm{SB}_{1}}\right)\right\|_{\infty} \leq 2 \sum_{j=r+1}^{n} \bar{\sigma}_{j}$.

Therefore, $\left\|\mathbf{G}(z)-\mathbf{G}_{\mathrm{tr}}(z)\right\|_{\infty} \leq 2\left\|\overline{\mathbf{L}}_{\mathrm{SB}}\right\|\left\|\overline{\mathbf{K}}_{\mathrm{SB}}\right\| \sum_{j=r+1}^{n} \bar{\sigma}_{j}$.

Corollary 1 Theorem 1 holds true subject to the following rank conditions $\operatorname{rank}\left[\overline{\mathbf{B}}_{\mathrm{SB}} \overline{\mathbf{B}}\right]=\operatorname{rank}\left[\overline{\mathbf{B}}_{\mathrm{SB}}\right]$ and $\operatorname{rank}\left[\begin{array}{c}\overline{\mathbf{C}}_{\mathrm{SB}} \\ \overline{\mathbf{C}}\end{array}\right]=\operatorname{rank}\left[\overline{\mathbf{C}}_{\mathrm{SB}}\right]$ (which follows from [16]) are satisfied.

Remark 6 The rank condition follows from [12].

Remark 7 When input and output related matrices $\overline{\mathbf{X}}_{\mathrm{WZ}} \geq 0$ and $\overline{\mathbf{Y}}_{\mathrm{WZ}} \geq 0$, respectively, then $\overline{\mathbf{P}}_{\mathrm{WZ}}=\overline{\mathbf{P}}_{\mathrm{E}}=\overline{\mathbf{P}}_{\mathrm{SB}}$ and $\overline{\mathbf{Q}}_{\mathrm{WZ}}=\overline{\mathbf{Q}}_{\mathrm{E}}=\overline{\mathbf{Q}}_{\mathrm{SB}}$. Otherwise, $\overline{\mathbf{P}}_{\mathrm{WZ}}<\overline{\mathbf{P}}_{\mathrm{SB}}$ and $\overline{\mathbf{Q}}_{\mathrm{WZ}}<\overline{\mathbf{Q}}_{\mathrm{SB}}$. Moreover, frequency limited Hankel values satisfy : $\left(\bar{\lambda}_{j}\left[\overline{\mathbf{P}}_{\mathrm{WZ}} \overline{\mathbf{Q}}_{\mathrm{WZ}}\right]\right)^{1 / 2} \leq\left(\bar{\lambda}_{j}\left[\overline{\mathbf{P}}_{\mathrm{SB}} \overline{\mathbf{Q}}_{\mathrm{SB}}\right]\right)^{1 / 2}$.

Remark 8 When the input and the output related matrices $\overline{\mathbf{X}}_{\mathrm{WZ}} \geq 0$ and $\overline{\mathbf{Y}}_{\mathrm{WZ}} \geq 0$, respectively, then approximated models obtained using [14] and proposed technique are the equivalent.

Theorem 2 The following Lyapunov equation for the proposed technique holds

$$
\begin{aligned}
\mathbf{A} \overline{\mathbf{P}}_{(\mathrm{ext})} \mathbf{A}^{\mathrm{T}}-\overline{\mathbf{P}}_{(\mathrm{ext})}+\overline{\mathbf{B}}_{(\mathrm{ext})} \overline{\mathbf{B}}_{(\mathrm{ext})}^{\mathrm{T}}=0, \\
\mathbf{A}^{\mathrm{T}} \overline{\mathbf{Q}}_{(\mathrm{ext})} \mathbf{A}-\overline{\mathbf{Q}}_{(\mathrm{ext})}+\overline{\mathbf{C}}_{(\mathrm{ext})}^{\mathrm{T}} \overline{\mathbf{C}}_{(\mathrm{ext})}=0 .
\end{aligned}
$$

Proof: Using (11), (13), (24) and (25) we have following

$$
\begin{aligned}
\overline{\mathbf{S}}_{\mathrm{WZ}} & =\operatorname{diag}\left[\overline{\mathbf{S}}_{\mathrm{WZ}}, \overline{\mathbf{S}}_{\mathrm{WZ}}\right]=\operatorname{diag}\left[\left(\bar{s}_{1}, . ., \bar{s}_{q-1}\right),\left(\bar{s}_{q}, . ., \bar{s}_{n}\right)\right](30) \\
\overline{\mathbf{S}}_{\mathrm{SB}} & =\operatorname{diag}\left[\overline{\mathbf{S}}_{\mathrm{WZ}} \overline{\mathbf{S}}_{\mathrm{SB}_{2}}\right]=\operatorname{diag}\left[\left(\bar{s}_{1}, \ldots, \bar{s}_{q-1}\right),\left(\hat{s}_{q}, \ldots, \hat{s}_{n}\right)\right](31) \\
\overline{\mathbf{R}}_{\mathrm{WZ}} & =\operatorname{diag}\left[\overline{\mathbf{R}}_{\mathrm{WZ}}, \overline{\mathbf{R}}_{\mathrm{WZ}}\right]=\operatorname{diag}\left[\left(\bar{r}_{1}, . ., \bar{r}_{k-1}\right),\left(\bar{r}_{k}, . ., \bar{r}_{n}\right)\right](32) \\
\overline{\mathbf{R}}_{\mathrm{SB}} & =\operatorname{diag}\left[\overline{\mathbf{R}}_{\mathrm{WZ}_{1}}, \overline{\mathbf{R}}_{\mathrm{SB}_{2}}\right]=\operatorname{diag}\left[\left(\bar{r}_{1}, . ., \bar{r}_{k-1}\right),\left(\hat{r}_{k}, . ., \hat{r}_{n}\right)\right](33)
\end{aligned}
$$

$\overline{\mathbf{S}}_{(\mathrm{ext})}$ and $\overline{\mathbf{R}}_{\text {(ext) }}$ are obtained by subtracting $(31-30)$ and $(33-32)$ respectively

$$
\overline{\mathbf{S}}_{(\mathrm{ext})}=\left[\begin{array}{cc}
0 & 0 \\
0 & \overline{\mathbf{S}}_{(\mathrm{ext})_{2}}
\end{array}\right], \quad \overline{\mathbf{R}}_{(\mathrm{ext})}=\left[\begin{array}{cc}
0 & 0 \\
0 & \overline{\mathbf{R}}_{(\mathrm{ext})_{2}}
\end{array}\right]
$$

where $\overline{\mathbf{S}}_{(\mathrm{ext})_{2}}=\overline{\mathbf{S}}_{\mathrm{SB}_{2}}-\overline{\mathbf{S}}_{\mathrm{WZ}_{2}}$ and $\overline{\mathbf{R}}_{(\mathrm{ext})_{2}}=\overline{\mathbf{R}}_{\mathrm{SB}_{2}}-\overline{\mathbf{R}}_{\mathrm{WZ}_{2}}$. $\overline{\mathbf{B}}_{\text {(ext) }}$ and $\overline{\mathbf{C}}_{\text {(ext) }}$ are obtained by subtracting $(24-12)$ and $(25-14)$ respectively

$$
\begin{aligned}
& \overline{\mathbf{B}}_{(\mathrm{ext})}=\overline{\mathbf{U}}_{(\mathrm{ext})}\left[\begin{array}{cc}
0 & 0 \\
0 & \overline{\mathbf{S}}_{(\mathrm{ext})_{2}}^{1 / 2}
\end{array}\right]=\overline{\mathbf{U}}_{(\mathrm{ext})} \overline{\mathbf{S}}_{(\mathrm{ext})}^{1 / 2}, \\
& \overline{\mathbf{C}}_{(\mathrm{ext})}=\left[\begin{array}{cc}
0 & 0 \\
0 & \overline{\mathbf{R}}_{(\mathrm{ext})_{2}}^{1 / 2}
\end{array}\right] \overline{\mathbf{V}}_{(\mathrm{ext})}^{\mathrm{T}}=\overline{\mathbf{R}}_{(\mathrm{ext})}^{1 / 2} \overline{\mathbf{V}}_{(\mathrm{ext})}^{\mathrm{T}}
\end{aligned}
$$

where $\overline{\mathbf{U}}_{(\mathrm{ext})}=\overline{\mathbf{U}}_{\mathrm{SB}}=\overline{\mathbf{U}}_{\mathrm{WZ}}$ and $\overline{\mathbf{V}}_{\mathrm{ext}}=\overline{\mathbf{V}}_{\mathrm{SB}}=\overline{\mathbf{V}}_{\mathrm{WZ}}$. Since,

$$
\begin{aligned}
\overline{\mathbf{X}}_{(\mathrm{ext})} & =\overline{\mathbf{B}}_{(\mathrm{ext})} \overline{\mathbf{B}}_{(\mathrm{ext})}^{\mathrm{T}}=\overline{\mathbf{U}}_{(\mathrm{ext})} \overline{\mathbf{S}}_{(\mathrm{ext})}^{1 / 2} \overline{\mathbf{S}}_{(\mathrm{ext})}^{1 / 2} \overline{\mathbf{U}}_{(\mathrm{ext})}^{\mathrm{T}} \\
& =\overline{\mathbf{U}}_{(\mathrm{ext})} \overline{\mathbf{S}}_{(\mathrm{ext})} \overline{\mathbf{U}}_{(\mathrm{ext})}^{\mathrm{T}}=\overline{\mathbf{U}}_{(\mathrm{ext})}\left(\overline{\mathbf{S}}_{\mathrm{SB}}-\overline{\mathbf{S}}_{\mathrm{WZ}}\right) \overline{\mathbf{U}}_{(\mathrm{ext})}^{\mathrm{T}} \\
& =\overline{\mathbf{U}}_{(\mathrm{ext})} \overline{\mathbf{S}}_{\mathrm{SB}} \overline{\mathbf{U}}_{(\mathrm{ext})}^{\mathrm{T}}-\overline{\mathbf{U}}_{(\mathrm{ext})} \overline{\mathbf{S}}_{\mathrm{WZ}} \overline{\mathbf{U}}_{(\mathrm{ext})}^{\mathrm{T}}=\overline{\mathbf{X}}_{\mathrm{SB}}-\overline{\mathbf{X}}_{\mathrm{WZ}},
\end{aligned}
$$

$$
\begin{aligned}
\overline{\mathbf{Y}}_{(\mathrm{ext})} & =\overline{\mathbf{C}}_{(\mathrm{ext})}^{\mathrm{T}} \overline{\mathbf{C}}_{(\mathrm{ext})}=\overline{\mathbf{V}}_{(\mathrm{ext})} \overline{\mathbf{R}}_{(\mathrm{ext})}^{1 / 2} \overline{\mathbf{R}}_{(\mathrm{ext})}^{1 / 2} \overline{\mathbf{V}}_{(\mathrm{ext})}^{\mathrm{T}} \\
& =\overline{\mathbf{V}}_{(\mathrm{ext})} \overline{\mathbf{R}}_{(\mathrm{ext})} \overline{\mathbf{V}}_{\text {(ext) }}^{\mathrm{T}}=\overline{\mathbf{V}}_{(\mathrm{ext})}\left(\overline{\mathbf{R}}_{\mathrm{SB}}-\overline{\mathbf{R}}_{\mathrm{WZ}}\right) \overline{\mathbf{V}}_{(\mathrm{ext})}^{\mathrm{T}} \\
& =\overline{\mathbf{V}}_{(\mathrm{ext})} \overline{\mathbf{R}}_{\mathrm{SB}} \overline{\mathbf{V}}_{(\mathrm{ext})}^{\mathrm{T}}-\overline{\mathbf{V}}_{\mathbf{\mathbf { R }}} \overline{\mathbf{R}}_{\mathrm{WZ}} \overline{\mathbf{V}}_{(\mathrm{ext})}^{\mathrm{T}}=\overline{\mathbf{Y}}_{\mathrm{SB}}-\overline{\mathbf{Y}}_{\mathrm{WZ}},
\end{aligned}
$$

using (9 and 20) in (34) and (10 and 21) in (35) we have following

$$
\begin{array}{r}
\left(\mathbf{A} \overline{\mathbf{P}}_{\mathrm{SB}} \mathbf{A}^{\mathrm{T}}-\overline{\mathbf{P}}_{\mathrm{SB}}\right)-\left(\mathbf{A} \overline{\mathbf{P}}_{\mathrm{WZ}} \mathbf{A}^{\mathrm{T}}-\overline{\mathbf{P}}_{\mathrm{WZ}}\right)=-\overline{\mathbf{X}}_{(\mathrm{ext})}, \\
\left(\mathbf{A}^{\mathrm{T}} \overline{\mathbf{Q}}_{\mathrm{SB}} \mathbf{A}-\overline{\mathbf{Q}}_{\mathrm{SB}}\right)-\left(\mathbf{A}^{\mathrm{T}} \overline{\mathbf{Q}}_{\mathrm{WZ}} \mathbf{A}-\overline{\mathbf{Q}}_{\mathrm{WZ}}\right)=-\overline{\mathbf{Y}}_{(\mathrm{ext})}, \\
\mathbf{A}\left(\overline{\mathbf{P}}_{\mathrm{SB}}-\overline{\mathbf{P}}_{\mathrm{WZ}}\right) \mathbf{A}^{\mathrm{T}}-\left(\overline{\mathbf{P}}_{\mathrm{SB}}-\overline{\mathbf{P}}_{\mathrm{WZ}}\right)=-\overline{\mathbf{X}}_{(\mathrm{ext})}, \\
\mathbf{A}^{\mathrm{T}}\left(\overline{\mathbf{Q}}_{\mathrm{SB}}-\overline{\mathbf{Q}}_{\mathrm{WZ}}\right) \mathbf{A}-\left(\overline{\mathbf{Q}}_{\mathrm{SB}}-\overline{\mathbf{Q}}_{\mathrm{WZ}}\right)=-\overline{\mathbf{Y}}_{(\mathrm{ext})},
\end{array}
$$

If controllability Gramian $\overline{\mathbf{P}}_{(\mathrm{ext})}=\overline{\mathbf{P}}_{\mathrm{SB}}-\overline{\mathbf{P}}_{\mathrm{WZ}}$ and observability Gramian $\overline{\mathbf{Q}}_{(\mathrm{ext})}=\overline{\mathbf{Q}}_{\mathrm{SB}}-\overline{\mathbf{Q}}_{\mathrm{WZ}}$ then

$$
\begin{aligned}
\mathbf{A} \overline{\mathbf{P}}_{(\mathrm{ext})} \mathbf{A}^{\mathrm{T}}-\overline{\mathbf{P}}_{(\mathrm{ext})}+\overline{\mathbf{B}}_{(\mathrm{ext})} \overline{\mathbf{B}}_{(\mathrm{ext})}^{\mathrm{T}}=0, \\
\mathbf{A}^{\mathrm{T}} \overline{\mathbf{Q}}_{(\mathrm{ext})} \mathbf{A}-\overline{\mathbf{Q}}_{(\mathrm{ext})}+\overline{\mathbf{C}}_{(\mathrm{ext})}^{\mathrm{T}} \overline{\mathbf{C}}_{(\mathrm{ext})}=0 .
\end{aligned}
$$

Corollary 2 Theorem 2 holds true subject to the realization $\left(\mathbf{A}, \overline{\mathbf{B}}_{(\mathrm{ext})}, \overline{\mathbf{C}}_{(\mathrm{ext})}, \mathbf{D}\right)$ is minimal and stable.

Remark 9 For the realization $\left\{\mathbf{A}, \overline{\mathbf{B}}_{(\mathrm{ext})}, \overline{\mathbf{C}}_{(\mathrm{ext})}, \mathbf{D}\right\}$ to the following Lyapunov equation

$$
\begin{array}{r}
\mathbf{A} \overline{\mathbf{P}}_{(\mathrm{ext})} \mathbf{A}^{\mathrm{T}}-\overline{\mathbf{P}}_{(\mathrm{ext})}+\overline{\mathbf{B}}_{(\mathrm{ext})} \overline{\mathbf{B}}_{(\mathrm{ext})}^{\mathrm{T}}=0, \\
\mathbf{A}^{\mathrm{T}} \overline{\mathbf{Q}}_{(\mathrm{ext})} \mathbf{A}-\overline{\mathbf{Q}}_{(\mathrm{ext})}+\overline{\mathbf{C}}_{(\mathrm{ext})}^{\mathrm{T}} \overline{\mathbf{C}}_{(\mathrm{ext})}=0
\end{array}
$$

where the input matrix $\overline{\mathbf{B}}_{\text {(ext) }} \geq 0$ and the output matrix $\overline{\mathbf{C}}_{(\text {ext }} \geq 0$ ensure positive (semi-positive) definiteness of the input and the output related matrices $\overline{\mathbf{B}}_{\mathrm{SB}}$ and $\overline{\mathbf{C}}_{\mathrm{SB}}$, respectively; consequently, positive definiteness of $\overline{\mathbf{P}}_{(\mathrm{ext})}$ and $\overline{\mathbf{Q}}_{(\mathrm{ext})}$ in a way leads to positive definiteness of $\overline{\mathbf{P}}_{\mathrm{SB}}$ and $\overline{\mathbf{Q}}_{\mathrm{SB}}$. 
Remark 10 Since the input matrix $\overline{\mathbf{B}}_{\mathrm{SB}}$ and the output matrix $\overline{\mathbf{C}}_{\mathrm{SB}}$ ensure positive (semi-positive) definiteness of the input and the output related matrices; consequently, positive definiteness of $\overline{\mathbf{P}}_{\mathrm{SB}}$ and $\overline{\mathbf{Q}}_{\mathrm{SB}}$ in a way leads to transformation matrix $\overline{\mathbf{T}}_{\mathrm{SB}}$, which subsequently, results in the stability preserving model order reduction technique. Moreover, $\overline{\mathbf{L}}_{\mathrm{SB}}$ and $\overline{\mathbf{K}}_{\mathrm{SB}}$ form bases for the derivation of a priori error bound for proposed technique.

Remark 11 WZ's technique [14] involves the computation of the similarity transformation matrix $\overline{\mathbf{T}}_{\mathrm{WZ}}$ employing the usage of the controllability and the observability Gramians $\overline{\mathbf{P}}_{\mathrm{WZ}}$ and $\overline{\mathbf{Q}}_{\mathrm{WZ}}$, respectively, are directly computed from the original system realization $\{\mathbf{A}, \mathbf{B}, \mathbf{C}\}$. Consequently, it requires $2 \times n^{\text {th }}$ number of Lyapunov equations for $n^{\text {th }}$ order systems to perform MOR; however, stability of the ROM obtained is not guaranteed. Whereas, the similarity transformation matrix $\overline{\mathbf{T}}_{\mathrm{SB}}$ obtained by using the proposed technique employing the usage of controllability and observability Gramians $\overline{\mathbf{P}}_{\mathrm{SB}}=\overline{\mathbf{P}}_{\mathrm{WZ}}+\overline{\mathbf{P}}_{(\mathrm{ext})}$ and $\overline{\mathbf{Q}}_{\mathrm{SB}}=\overline{\mathbf{Q}}_{\mathrm{WZ}}+\overline{\mathbf{Q}}_{(\mathrm{ext})}$, respectively, are computed from the realization $\left\{\mathbf{A}, \overline{\mathbf{B}}_{\mathrm{SB}}, \overline{\mathbf{C}}_{\mathrm{SB}}\right\}$. The relationship between Gramians matrices of WZ [14] and the proposed technique is given as:

$$
\begin{array}{ll}
\mathbf{A}\left(\overline{\mathbf{P}}_{\mathrm{WZ}}+\overline{\mathbf{P}}_{(\mathrm{ext})}\right) \mathbf{A}^{\mathrm{T}}-\left(\overline{\mathbf{P}}_{\mathrm{WZ}}+\overline{\mathbf{P}}_{(\mathrm{ext})}\right) & \\
+\left(\overline{\mathbf{X}}_{\mathrm{WZ}}+\overline{\mathbf{X}}_{(\mathrm{ext})}\right)=0, & \text { for } \bar{s}_{n}<0, \\
\mathbf{A} \overline{\mathbf{P}}_{\mathrm{WZ}} \mathbf{A}^{\mathrm{T}}-\overline{\mathbf{P}}_{\mathrm{WZ}}+\overline{\mathbf{X}}_{\mathrm{en}}=0, & \text { for } \bar{s}_{n} \geq 0, \\
\mathbf{A}^{\mathrm{T}}\left(\overline{\mathbf{Q}}_{\mathrm{WZ}}+\overline{\mathbf{Q}}_{(\mathrm{ext})}\right) \mathbf{A}-\left(\overline{\mathbf{Q}}_{\mathrm{WZ}}+\overline{\mathbf{Q}}_{(\mathrm{ext})}\right) & \\
+\left(\overline{\mathbf{Y}}_{\mathrm{WZ}}+\overline{\mathbf{Y}}_{(\mathrm{ext})}\right)=0, & \text { for } \bar{r}_{n}<0, \\
\mathbf{A}^{\mathrm{T}} \overline{\mathbf{Q}}_{\mathrm{WZ}} \mathbf{A}-\overline{\mathbf{Q}}_{\mathrm{WZ}}+\overline{\mathbf{Y}}_{\mathrm{WZ}}=0, & \text { for } \bar{r}_{n} \geq 0, \\
\mathbf{A} \overline{\mathbf{P}}_{(\mathrm{ext})} \mathbf{A}^{\mathrm{T}}-\overline{\mathbf{P}}_{(\mathrm{ext})}+\overline{\mathbf{X}}_{(\mathrm{ext})}=0, & \text { for } \bar{s}_{n}<0, \\
\mathbf{A}^{\mathrm{T}} \overline{\mathbf{Q}}_{(\mathrm{ext})} \mathbf{A}-\overline{\mathbf{Q}}_{(\mathrm{ext})}+\overline{\mathbf{Y}}_{(\mathrm{ext})}=0, & \text { for } \bar{r}_{n}<0 .
\end{array}
$$

Since

$$
\begin{array}{rlr}
\overline{\mathbf{X}}_{\mathrm{SB}} & =\overline{\mathbf{U}}_{\mathrm{SB}}\left(\overline{\mathbf{S}}_{\mathrm{SB}}\right)^{1 / 2}\left(\overline{\mathbf{S}}_{\mathrm{SB}}\right)^{1 / 2} \overline{\mathbf{U}}_{\mathrm{SB}}^{\mathrm{T}} \\
& =\overline{\mathbf{X}}_{\mathrm{WZ}}+\overline{\mathbf{X}}_{(\mathrm{ext})}, \text { for } \bar{s}_{n}<0, \\
\overline{\mathbf{X}}_{\mathrm{SB}} & =\overline{\mathbf{U}}_{\mathrm{SB}}\left(\overline{\mathbf{S}}_{\mathrm{SB}}\right)^{1 / 2}\left(\overline{\mathbf{S}}_{\mathrm{SB}}\right)^{1 / 2} \overline{\mathbf{U}}_{\mathrm{SB}}^{\mathrm{T}} \\
& =\overline{\mathbf{X}}_{\mathrm{WZ}}, \quad \text { for } \bar{s}_{n} \geq 0, \\
\overline{\mathbf{Y}}_{\mathrm{SB}} & =\overline{\mathbf{V}}_{\mathrm{SB}}^{\mathrm{T}}\left(\overline{\mathbf{R}}_{\mathrm{SB}}\right)^{1 / 2}\left(\overline{\mathbf{R}}_{\mathrm{SB}}\right)^{1 / 2} \overline{\mathbf{V}}_{\mathrm{SB}} \\
& =\overline{\mathbf{Y}}_{\mathrm{WZ}}+\overline{\mathbf{Y}}_{(\mathrm{ext})}, \quad \text { for } \bar{r}_{n}<0, \\
\overline{\mathbf{Y}}_{\mathrm{SB}} & =\overline{\mathbf{V}}_{\mathrm{SB}}^{\mathrm{T}}\left(\overline{\mathbf{R}}_{\mathrm{SB}}\right)^{1 / 2}\left(\overline{\mathbf{R}}_{\mathrm{SB}}\right)^{1 / 2} \overline{\mathbf{V}}_{\mathrm{SB}} \\
& =\overline{\mathbf{Y}}_{\mathrm{WZ}}, \quad \text { for } \bar{r}_{n} \geq 0 .
\end{array}
$$

Consequently, the proposed method requires $4 \times n^{\text {th }}$ number of Lyapunov equations to obtained ROM and the stability is also guaranteed.

\section{Numerical Simulations}

In this section, a comparison among different techniques is presented. Reduced-order transfer functions and poles location of ( [14-16]) and the proposed technique are also provided where [14] produces unstable ROMs. Figures 1 and 2 represent the frequency response Bode plot (magnitude and phase) in the given frequency range. Furthermore, Figures 3 and 5 represent the frequency response error in the entire frequency range of the approximated model obtained by using existing ( [14-16]) and proposed techniques, whereas, Figures 4 and 6 signify the frequency response errors in the given limited discrete-frequency ranges of the approximated model in the given frequency interval acquired via using existing and proposed techniques. Tables 2, 3 and 4 provide the ROMs obtained by using existing ( [14-16]) and proposed techniques; whereas, the Table 5 provide the location of poles by using the WZ [14] proposed technique; furthermore, the Table 6 provides the error bounds by using existing ( [14-16]) and proposed techniques.

Example 1 Benchmark example of $48^{\text {th }}$ order is presented here, the building model (the Los Angeles University Hospital) [18], it contains 8 floors each have 3 degrees of freedom, namely displacement in two different directions $x_{1}$ and $x_{2}$, and rotation. State-space form representation of given an example is available at [19], given model is discretized at sampling time $T_{\mathrm{s}}=0.001 \mathrm{~s}$, with the given frequency interval $\left[\bar{\omega}_{1}-\bar{\omega}_{2}\right]=[0.01 \pi-0.25 \pi] \mathrm{rad} / \mathrm{s}$, where $\mathbf{G}_{\mathrm{tr}}(z)$ is the approximated model of $4^{\text {th }}$ order obtained by using [14-16] and proposed techniques. Figure 1 provides a comparison for the frequency response Bode plot (magnitude, phase) in the given frequency range $[0.01 \pi-0.25 \pi] \mathrm{rad} / \mathrm{s}$. Reducedorder transfer functions and poles location of ( [14-16]) and proposed techniques are also provided in the Tables 3 and 5, respectively, poles location of ROM obtained by using [14] are $z=0.9945 \pm 0.0522 \mathrm{i}, 0.9860 \pm 0.1340 \mathrm{i}$. Whereas, poles location of ROM obtained by using the proposed technique are $z=0.9946 \pm 0.0525 \mathrm{i}, 0.9862 \pm 0.1348 \mathrm{i}$. Table 6 provide a comparison for the frequency response error bounds in the given frequency range $[0.01 \pi-0.25 \pi] \mathrm{rad} / \mathrm{s}$. Furthermore, the proposed algorithm grants stable ROMs, minimum frequency response error along with the frequency response error bound comparable with different existing stability retaining algorithms ( [14-16]) in the given frequency range.

\begin{tabular}{|c|c|}
\hline \multirow{2}{*}{ Techniques } & Reduced order model $\mathbf{G}_{\mathrm{tr}}(z)$ \\
\cline { 2 - 2 } & $\mathbf{4}^{\text {th }}$ Order ROMs \\
\hline WZ [14] & $\frac{7.69 \mathrm{e}^{-5} z^{3}-0.0002306 z^{2}+0.0002313 z-7.759 \mathrm{e}^{-5}}{z^{4}-3.961 z^{3}+5.905 z^{2}-3.926 z+0.9821}$ \\
\hline GSI [15] & $\frac{7.781 \mathrm{e}^{-5} z^{3}-0.0002332 z^{2}+0.0002338 z-7.843 \mathrm{e}^{-5}}{z^{4}-3.961 z^{3}+5.904 z^{2}-3.925 z+0.9818}$ \\
\hline GSII [15] & $\frac{7.773 \mathrm{e}^{-5} z^{3}-0.000233 z^{2}+0.0002336 z-7.836 \mathrm{e}^{-5}}{z^{4}-3.961 z^{3}+5.904 z^{2}-3.925 z+0.9818}$ \\
\hline IG [16] & $\frac{6.283 \mathrm{e}^{-5} z^{3}-0.0001877 z^{2}+0.0001875 z-6.266 \mathrm{e}^{-5}}{z^{4}-3.966 z^{3}+5.919 z^{2}-3.941 z+0.9874}$ \\
\hline Proposed & $\frac{7.673 \mathrm{e}^{-5} z^{3}-0.0002296 z^{2}+0.0002297 z-7.693 \mathrm{e}^{-5}}{z^{4}-3.962 z^{3}+5.906 z^{2}-3.928 z+0.9829}$ \\
\hline
\end{tabular}

Tab. 2. Reduced order models for Example-1. 
Example 2 Consider a $20^{\text {th }}$ order band-stop Butterworth filter with normalized edge frequencies ranges [0.5 $0.6] \mathrm{rad} / \mathrm{sample}$, with following transfer function form (36) [under this paragraph], with the given frequency interval $\left[\bar{\omega}_{1}-\bar{\omega}_{2}\right]=[0.5 \pi-0.9 \pi] \mathrm{rad} / \mathrm{s}$, where $\mathbf{G}_{\mathrm{tr}}(z)$ is the approximated model of $7^{\text {th }}$ order obtained by using [14-16] and proposed techniques. Figure 2 provides a comparison for the frequency response Bode plot (magnitude, phase) in the given frequency range $[0.5 \pi-0.9 \pi] \mathrm{rad} / \mathrm{s}$. Reduced-order transfer functions and poles location of ( [14-16]) and proposed techniques are also provided in the Tabs. 3 and 5, respectively, poles location of ROM obtained by using [14] are $z=$ $-0.0910,-0.2659 \pm 0.9534 \mathrm{i},-0.1738 \pm 0.9693 \mathrm{i},-0.0472 \pm$ $0.9770 \mathrm{i}$. Whereas, poles location of ROM obtained by using the proposed technique are $z=-0.1309,-0.2770 \pm$ $0.9513 \mathrm{i},-0.1845 \pm 0.9671 \mathrm{i},-0.0673 \pm 0.9874 \mathrm{i}$. Table 6 provide a comparison for the frequency response error bounds in the given frequency range $[0.5 \pi-0.9 \pi] \mathrm{rad} / \mathrm{s}$. Furthermore, the proposed algorithm grants stable ROMs, minimum frequency response error along with the frequency response error bound comparable with different existing stability retaining algorithms ( [14-16]) in the given frequency range.
Example 3 Consider a $6^{\text {th }}$ order stable discrete-time system [16] having following state space representation (37), with the given frequency interval $\left[\bar{\omega}_{1}-\bar{\omega}_{2}\right]=[0.65 \pi-0.81 \pi]$ $\mathrm{rad} / \mathrm{s}$. Figures 3 and 5 provide a comparison for the frequency response error $\bar{\sigma}\left[\mathbf{G}(z)-\mathbf{G}_{\mathrm{tr}}(z)\right]$ for the entire frequency interval, where $\mathbf{G}_{\mathrm{tr}}(z)$ are approximated models of $4^{\text {th }}$ and $5^{\text {th }}$ order respectively obtained by using [14-16] and proposed techniques. Figures 4 and 6 provide a comparison for the frequency response error $\bar{\sigma}\left[G(z)-G_{\text {tr }}(z)\right]$ in the given frequency range $[0.65 \pi-0.81 \pi] \mathrm{rad} / \mathrm{s}$. Reduced order transfer functions and poles location of ( [14-16]) and proposed techniques are also provided in the Tabs. 4 and 5, respectively, [14] produces unstable $4^{\text {th }}$ and $5^{\text {th }}$ order ROMs with poles location at $z=-2.5368,-0.3400,-0.3721 \pm 0.8901 \mathrm{i}$, and $z=2.2355,-0.0368 \pm 1.1440 \mathrm{i},-0.0996 \pm 0.7056 \mathrm{i}$ respectively. Whereas, the proposed technique produces stable $4^{\text {th }}$ and $5^{\text {th }}$ order ROMs with poles location at $z=$ $0.0139 \pm 0.9454 \mathrm{i}, 0.3842 \pm 0.7131 \mathrm{i}$, and $z=0.5879,0.0015 \pm$ $0.9408 \mathrm{i}, 0.2884 \pm 0.7024 \mathrm{i}$, respectively. Table 6 provide a comparison for the frequency response error bounds in the given frequency range $[0.65 \pi-0.81 \pi] \mathrm{rad} / \mathrm{s}$. Furthermore, the proposed algorithm grants stable ROMs, minimum frequency response error along with the frequency response error bound comparable with the different existing stability retaining algorithms ( [14-16]) in the given frequency range.

$$
\begin{aligned}
& 3.62 \mathrm{e}^{-9} z^{20}-1.125 \mathrm{e}^{-23} z^{19}-3.62 \mathrm{e}^{-8} z^{18}+1.607 \mathrm{e}^{-22} z^{17}+1.629 \mathrm{e}^{-7} z^{16}+5.144 \mathrm{e}^{-23} z^{15}-4.344 \mathrm{e}^{-7} z^{14}-8.179 \mathrm{e}^{-21} z^{13} \\
& +7.601 \mathrm{e}^{-7} z^{12}-2.685 \mathrm{e}^{-20} z^{11}-9.122 \mathrm{e}^{-7} z^{10}-3.035 \mathrm{e}^{-20} z^{9}+7.601 \mathrm{e}^{-7} z^{8}-1.276 \mathrm{e}^{-20} z^{7}-4.344 \mathrm{e}^{-7} z^{6}-1.003 \mathrm{e}^{-21} z^{5} \\
& G(z)=\frac{+1.629 \mathrm{e}^{-7} z^{4}+3.215 \mathrm{e}^{-23} z^{3}-3.62 \mathrm{e}^{-8} z^{2}+3.62 \mathrm{e}^{-9}}{z^{20}+2.85 z^{19}+11.65 z^{18}+23.34 z^{17}+53.82 z^{16}+82.43 z^{15}+135.7 z^{14}+165.3 z^{13}+210.6 z^{12}+207.8 z^{11}+212.2 z^{10}} \\
& +169.9 z^{9}+140.8 z^{8}+90.38 z^{7}+60.64 z^{6}+30.11 z^{5}+16.07 z^{4}+5.688 z^{3}+2.321 z^{2}+0.4628 z+0.1328
\end{aligned}
$$

\begin{tabular}{|c|c|}
\hline \multirow{2}{*}{ Techniques } & Reduced order model $\mathbf{G}_{\mathrm{tr}(z)}$ \\
\hline & $7^{\text {th }}$ Order ROMs \\
\hline \multirow{2}{*}{ WZ [14] } & $3.62 \mathrm{e}^{-9} z^{7}+0.04341 z^{6}+0.00285 z^{5}+0.09095 z^{4}-0.02476 z^{3}+0.05937 z^{2}-0.0261 z+0.01779$ \\
\hline & $z^{7}+1.065 z^{6}+3.263 z^{5}+2.188 z^{4}+3.246 z^{3}+1.189 z^{2}+0.9917 z+0.08274$ \\
\hline \multirow{2}{*}{ GS-I [15] } & $0.01053 z^{6}-0.02528 z^{5}+0.01888 z^{4}-0.05901 z^{3}+0.02622 z^{2}-0.03108 z+0.0211$ \\
\hline & $z^{7}+1.033 z^{6}+3.264 z^{5}+2.172 z^{4}+3.288 z^{3}+1.235 z^{2}+1.028 z+0.1111$ \\
\hline \multirow{2}{*}{ GS-II [15] } & $3.62 \mathrm{e}^{-9} z^{7}-0.02857 z^{6}-0.0644 z^{5}-0.1308 z^{4}-0.1524 z^{3}-0.1503 z^{2}-0.08584 z-0.04303$ \\
\hline & $z^{7}+1.144 z^{6}+3.365 z^{5}+2.488 z^{4}+3.464 z^{3}+1.509 z^{2}+1.103 z+0.1826$ \\
\hline \multirow{2}{*}{ IG [16] } & $3.62 \mathrm{e}^{-9} z^{7}+0.01767 z^{6}-0.01624 z^{5}+0.01523 z^{4}-0.05923 z^{3}-0.01421 z^{2}-0.04263 z-0.006478$ \\
\hline & $z^{7}+1.152 z^{6}+3.37 z^{5}+2.458 z^{4}+3.44 z^{3}+1.419 z^{2}+1.073 z+0.1354$ \\
\hline \multirow{2}{*}{ Proposed } & $3.62 \mathrm{e}^{-9} z^{7}-0.008832 z^{6}-0.0339 z^{5}-0.07912 z^{4}-0.1026 z^{3}-0.1257 z^{2}-0.06966 z-0.04929$ \\
\hline & $z^{7}+1.189 z^{6}+3.398 z^{5}+2.52 z^{4}+3.458 z^{3}+1.426 z^{2}+1.064 z+0.122$ \\
\hline
\end{tabular}

$$
\left[\begin{array}{l|l}
\mathbf{A} & \mathbf{B} \\
\hline \mathbf{C} & \mathbf{D}
\end{array}\right]=\left[\begin{array}{cccccc|c}
1.4637 & -2.2838 & 2.0587 & -1.4467 & 0.6746 & -0.1825 & 1 \\
1 & 0 & 0 & 0 & 0 & 0 & 0 \\
0 & 1 & 0 & 0 & 0 & 0 & 0 \\
0 & 0 & 1 & 0 & 0 & 0 & 0 \\
0 & 0 & 0 & 1 & 0 & 0 & 0 \\
0 & 0 & 0 & 0 & 1 & 0 & 0 \\
\hline 0.0799 & 0.1351 & 0.2388 & 0.1370 & 0.0776 & -0.0011 & 0.0107
\end{array}\right]
$$

Tab. 3. Reduced order models for Example-2. 


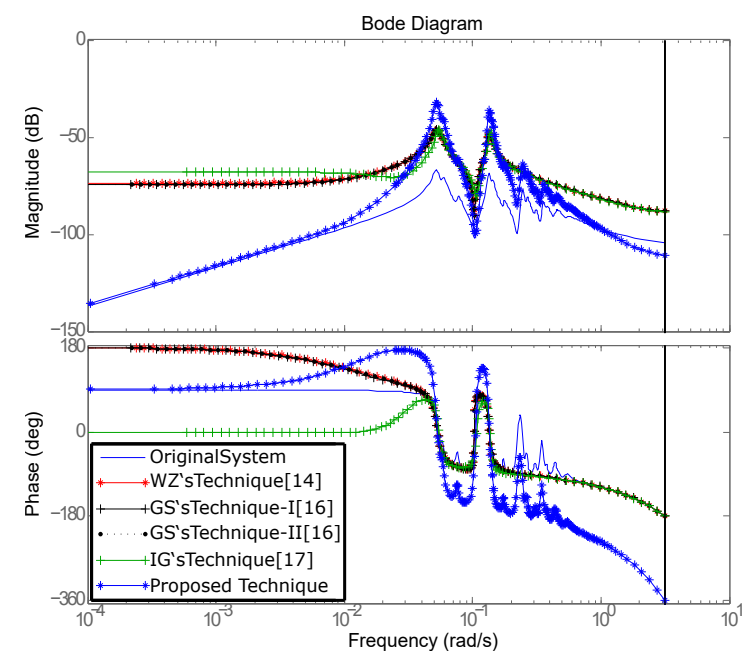

Fig. 1. Frequency response Bode plot (magnitude and phase) in the given frequency range $\left[\bar{\omega}_{1}-\bar{\omega}_{2}\right]=$ $[0.01 \pi-0.25 \pi] \mathrm{rad} / \mathrm{s}$ of $4^{\text {th }}$ order for Example- 1 .

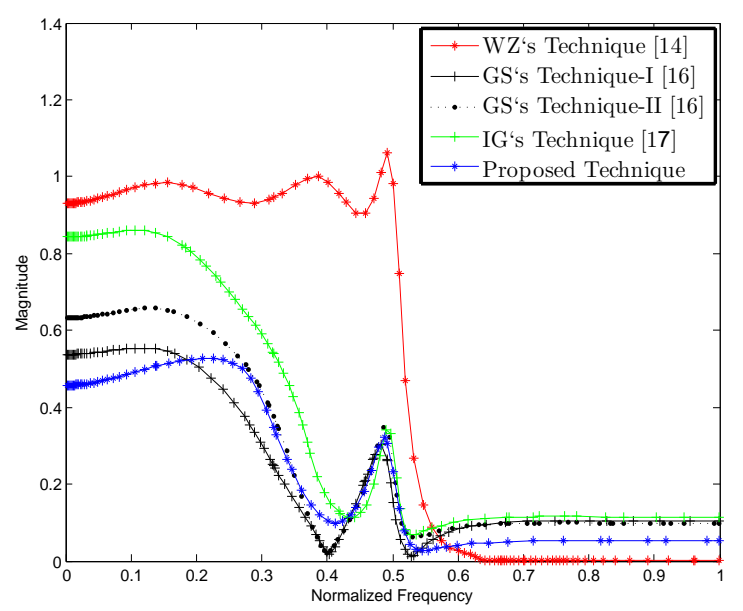

Fig. 3. Frequency response error $\bar{\sigma}\left[\mathbf{G}(z)-\mathbf{G}_{\mathrm{tr}}(z)\right]$ comparison of $4^{\text {th }}$ order for Example-3.

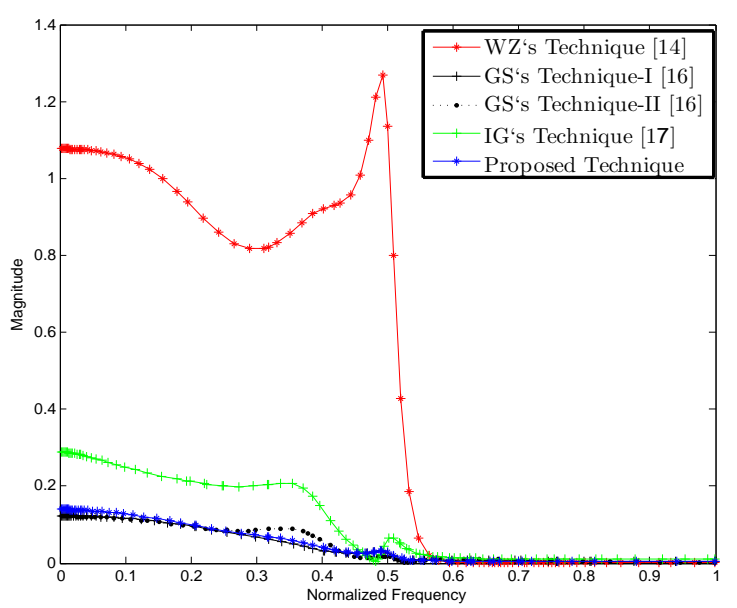

Fig. 5. Frequency response error $\bar{\sigma}\left[\mathbf{G}(z)-\mathbf{G}_{\mathrm{tr}}(z)\right]$ comparison of $5^{\text {th }}$ order for Example-3.

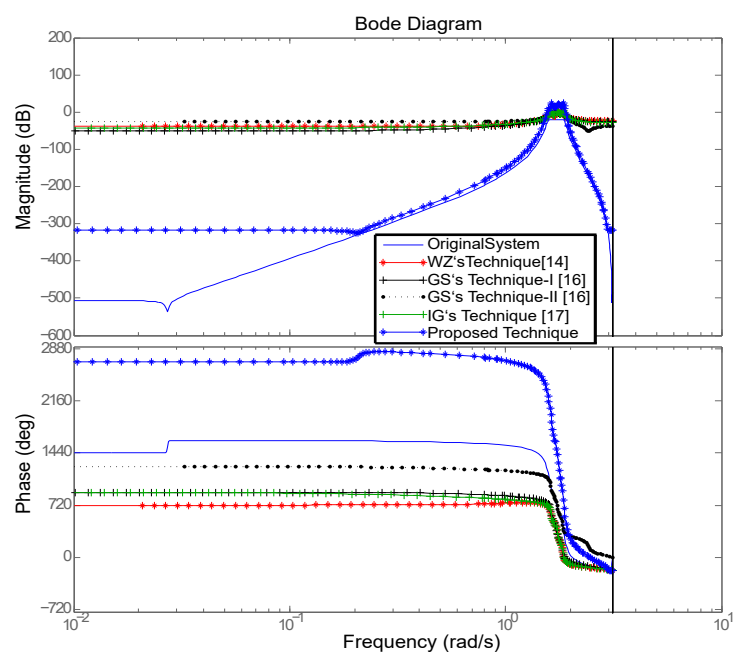

Fig. 2. Frequency response Bode plot (magnitude and phase) in the given frequency range $\left[\bar{\omega}_{1}-\bar{\omega}_{2}\right]=$ $[0.5 \pi-0.9 \pi] \mathrm{rad} / \mathrm{s}$ of $7^{\text {th }}$ order for Example- 2 .

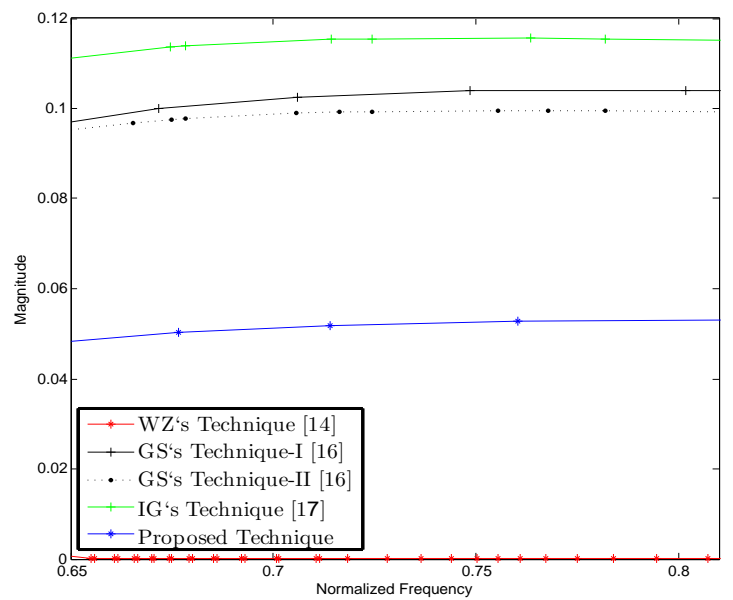

Fig. 4. $\bar{\sigma}\left[\mathbf{G}(z)-\mathbf{G}_{\mathrm{tr}}(z)\right]$ in the given frequency range $\left[\bar{\omega}_{1}-\bar{\omega}_{2}\right]=[0.65 \pi-0.81 \pi] \mathrm{rad} / \mathrm{s}$ of $4^{\text {th }}$ order for Example-3.

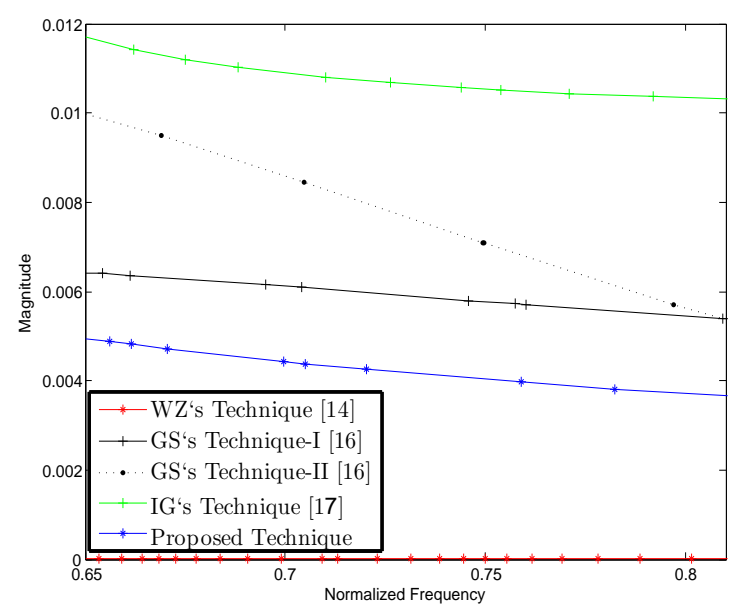

Fig. 6. $\bar{\sigma}\left[G(z)-G_{\mathrm{tr}}(z)\right]$ in the given frequency range $\left[\bar{\omega}_{1}-\bar{\omega}_{2}\right]=[0.65 \pi-0.81 \pi] \mathrm{rad} / \mathrm{s}$ of $5^{\text {th }}$ order for Example-3. 


\begin{tabular}{|c|c|c|}
\hline \multirow{2}{*}{ Techniques } & \multicolumn{2}{|c|}{ Reduced order model $\mathbf{G}_{\text {tr }}(z)$} \\
\cline { 2 - 3 } & $\mathbf{4}^{\text {th }}$ Order ROMs & $\mathbf{5}^{\text {th }}$ Order ROMs \\
\hline \multirow{2}{*}{ WZ [14] } & $\frac{0.0107 z^{4}+0.03384 z^{3}+0.04972 z^{2}+0.03774 z+0.01755}{z^{4}+3.621 z^{3}+3.934 z^{2}+3.319 z+0.8027}$ & $\frac{0.0107 z^{5}+0.06433 z^{4}+0.16 z^{3}+0.2176 z^{2}+0.1518 z+0.06955}{z^{5}-1.781 z^{4}+1.616 z^{3}-3.2 z^{2}+0.4466 z-1.296}$ \\
\hline \multirow{2}{*}{ GSI [15] } & $\frac{-0.01431 z^{3}+0.2195 z^{2}+0.08892 z+0.275}{z^{4}-0.8755 z^{3}+1.536 z^{2}-0.759 z+0.5415}$ & $\frac{0.07933 z^{4}+0.1585 z^{3}+0.2797 z^{2}+0.1945 z+0.1119}{z^{5}-1.149 z^{4}+1.776 z^{3}-1.341 z^{2}+0.7839 z-0.2889}$ \\
\hline \multirow{2}{*}{ GSII [15] } & $\frac{0.0107 z^{4}+0.02961 z^{3}+0.1995 z^{2}+0.1517 z+0.2255}{z^{4}-1.025 z^{3}+1.604 z^{2}-0.8832 z+0.5752}$ & $\frac{0.0107 z^{5}+0.06697 z^{4}+0.1765 z^{3}+0.2586 z^{2}+0.1979 z+0.09983}{z^{5}-1.18 z^{4}+1.804 z^{3}-1.382 z^{2}+0.8073 z-0.3008}$ \\
\hline \multirow{2}{*}{ IG [16] } & $\frac{0.0107 z^{4}-0.1022 z^{3}+0.1515 z^{2}-0.04009 z+0.1921}{z^{4}-0.6289 z^{3}+1.648 z^{2}-0.5681 z+0.666}$ & $\frac{0.0107 z^{5}+0.06833 z^{4}+0.1593 z^{3}+0.2539 z^{2}+0.1618 z+0.1061}{z^{5}-1.286 z^{4}+1.999 z^{3}-1.645 z^{2}+0.9858 z-0.4375}$ \\
\hline \multirow{2}{*}{ Proposed } & $\frac{0.0107 z^{4}-0.02853 z^{3}+0.2084 z^{2}+0.06448 z+0.2592}{z^{4}-0.7962 z^{3}+1.571 z^{2}-0.7052 z+0.5865}$ & $\frac{0.0107 z^{5}+0.06427 z^{4}+0.1814 z^{3}+0.2585 z^{2}+0.2048 z+0.1054}{z^{5}-1.168 z^{4}+1.804 z^{3}-1.373 z^{2}+0.8115 z-0.3}$ \\
\hline
\end{tabular}

Tab. 4. Reduced order models for Example-3.

\begin{tabular}{|l|l|l|l|}
\hline Examples & $\begin{array}{c}\text { Order } \\
\text { of ROMs }\end{array}$ & WZ's Technique [14] & Proposed Technique \\
\hline Example-1 & $4^{\text {th }}$ order & $0.9945 \pm 0.0522 \mathrm{i}, 0.9860 \pm 0.1340 \mathrm{i}$ & $0.9946 \pm 0.0525 \mathrm{i}, 0.9862 \pm 0.1348 \mathrm{i}$ \\
\hline Example-2 & $7^{\text {th }}$ order & $\begin{array}{l}-0.0910,-0.2659 \pm 0.9534 \mathrm{i}, \\
-0.1738 \pm 0.9693 \mathrm{i},-0.0472 \pm 0.9770 \mathrm{i}\end{array}$ & $-0.1309,-0.2770 \pm 0.9513 \mathrm{i}$, \\
& & $-0.1845 \pm 0.9671 \mathrm{i},-0.0673 \pm 0.9874 \mathrm{i}$ \\
\hline \multirow{2}{*}{ Example-3 3} & $4^{\text {th }}$ order & $-2.5368,-0.3400,-0.3721 \pm 0.8901 \mathrm{i}$ & $0.0139 \pm 0.9454 \mathrm{i}, 0.3842 \pm 0.7131 \mathrm{i}$ \\
\cline { 2 - 4 } & $5^{\text {th }}$ order & $2.2355,-0.0368 \pm 1.1440 \mathrm{i},-0.0996 \pm 0.7056 \mathrm{i}$ & $0.5879,0.0015 \pm 0.9408 \mathrm{i}, 0.2884 \pm 0.7024 \mathrm{i}$ \\
\hline
\end{tabular}

Tab. 5. Poles locations of reduced order models.

\begin{tabular}{|c|c|c|c|c|c|c|}
\hline Examples & $\begin{array}{c}\text { Order } \\
\text { of ROMs }\end{array}$ & BT [5] & GS-I [15] & GS-II [15] & IG [16] & Proposed \\
\hline Example-1 & $4^{\text {th }}$ order & 5.6190 & 5.8478 & 5.855 & 7.2905 & 5.8096 \\
\hline Example-2 & $7^{\text {th }}$ order & 24.115 & 25.022 & 25.064 & 24.937 & 24.884 \\
\hline \multirow{2}{*}{ Example-3 } & $4^{\text {th }}$ order & 39.268 & 45.163 & 43.832 & 46.163 & 41.991 \\
\cline { 2 - 7 } & $5^{\text {th }}$ order & 40.714 & 55.038 & 53.649 & 46.523 & 44.663 \\
\hline
\end{tabular}

Tab. 6. Theoretical and actual error bounds comparison.

\section{Analysis \& Discussion}

From Figs. 4 and 6 it is determined that truncated systems attained from WZ's [14] method give low approximation error as in contrast to the different existing techniques, however, it occasionally yields unstable truncated systems as proven in Tab. 5. Whereas, the different existing methods (GS-I [15], GS-II [15], IG [16]) produce stable ROMs and also provide the a priori error bound formula; however, these methods produce large approximation errors. The proposed approach produces stable ROMs, as proven in Tab. 5. Furthermore, Table 6 provides the comparison among theoretical error bound (BT [5]) and actual error bounds of existing discrete-frequency limited stability preserving approaches (GS-I [15], GS-II [15], IG [16]), and the proposed approach. It can be observed that as compared to the existing stability retaining techniques, the proposed method yields better approximation error along with the a priori error bound formula.

\section{Conclusion}

The frequency restricted improved MOR approach is proposed for the discrete-time systems. The proposed technique produces stable ROMs and lowers approximation error along with the formula for the a priori error bound calculation. MOR technique presented by $\mathrm{WZ}$ provides unstable ROMs and does not provide the a priori error bound formula, whereas the different existing methods produce stable ROMs and also provide the a priori error bound formula; however, these methods produce large approximation errors. The frequency response error of the proposed method is well comparable with the other existing methods. Numerical examples have proven that the proposed method provides stable ROMs, lower approximation error, and the a priori error bound formula, which shows the efficacy of the presented method. 


\section{Acknowledgments}

This research work was supported by MCS, NUST.

\section{References}

[1] ISLAM, S. N., DAS, S. Isosceles triangular resonator based compact triple band quad element multi terminal antenna. Radioengineering, 2020, vol. 29, no. 1, p. 52-58. DOI: 10.13164/re.2020.0052

[2] GALAJDA, P., SLOVAK, S., SOKOL, M., et al. Integrated M-sequence based transceiver for UWB sensor networks. Radioengineering, 2019, vol. 28, no. 1, p. 175-182. DOI: $10.13164 /$ re.2019.0175

[3] TIEN, N. H. A., KHA, H. H. Harvested energy and spectral efficiency trade-offs in multicell MIMO wireless networks. Radioengineering, 2019, vol. 28, no. 1, p. 331-339. DOI: 10.13164/re.2019.0331

[4] IMRAN, M., GHAFOOR, A. Frequency limited model reduction techniques with error bounds. IEEE Transactions on Circuits and Systems II: Express Briefs, 2017, vol. 10, no. 65, p. 86-90. DOI: 10.1109/TCSII.2017.2703117

[5] MOORE, B. C. Principal component analysis in linear systems:controllability, observability, and model reduction. IEEE Transactions on Automatic Control, 1981, vol. 26, no. 1, p. 17-32. DOI: 10.1109/TAC.1981.1102568

[6] ENNS, D. F. Model reduction with balanced realizations: An error bound and a frequency weighted generalization. In Proceedings of Conference on Decision and Control. Las Vegas (NV, USA), 1984, p. 127-132. DOI: 10.1109/CDC.1984.272286

[7] SREERAM, V., ANDERSON, B. D. O., MADIEVSKI, A. G. New results on frequency weighted balanced reduction technique. In Proceedings of American Control Conference (ACC). Seattle (WA, USA), 1995 p. 4004-4009. DOI: 10.1109/ACC.1995.532684

[8] LIN, C. A., CHIU, T. Y. Model reduction via frequency weighted balanced realization. In Proceedings of American Control Conference (ACC). San Diego (CA, USA), 1990, p. 2069-2070. DOI: $10.23919 /$ ACC. 1990.4791093

[9] WANG, G., SREERAM, V., LIU, W. Q. A new frequency weighted balanced truncation method and an error bound. IEEE Transactions on Automatic Control, 1999, vol. 44, no. 9, p. 1734-1737. DOI: $10.1109 / 9.788542$

[10] VARGA, A., ANDERSON, B. D. O. Accuracy-enhancing methods for balancing related frequency-weighted model and controller reduction. Automatica, 2003, vol. 39, no. 5, p. 919-927. DOI: $10.1016 / \mathrm{S} 0005-1098(03) 00030-\mathrm{X}$

[11] SREERAM, V. An improved frequency weighted balanced related technique with error bound. Proceedings of Conference on Decision and Control (CDC). Nassau (Bahamas), 2004, p. 3084-3089. DOI: $10.1109 /$ CDC.2004.1428940

[12] GHAFOOR, A., SREERAM, V. A survey/review of frequencyweighted balanced model reduction techniques. Journal of Dynamic Systems, Measurement and Control, 2008, vol. 130, no. 6, p. 1-16. DOI: $10.1115 / 1.2977468$
[13] IMRAN, M., GHAFOOR, A., SREERAM, V. A frequency weighted model order reduction technique and error bounds. Automatica, 2014, vol. 50, no. 12, p. 3304-3309. DOI: 10.1016/j.automatica.2014.10.062

[14] WANG, D., ZILOUCHIAN, A. Model reduction of discrete linear system via frequency domain balanced realization. IEEE Transactions on Circuits and Systems I: Fundamental Theory and Applications, 2000, vol. 47, no. 6, p. 830-837. DOI: 10.1109/81.852936

[15] GHAFOOR, A., SREERAM, V. Model reduction via limited frequency interval Gramians. IEEE Transactions on Circuits and Systems I: Regular Papers, 2008, vol. 55, no. 9, p. 2806-2812. DOI: 10.1109/TCSI.2008.920092

[16] IMRAN, M., GHAFOOR, A. Stability preserving model reduction technique and error bounds using frequency-limited Gramians for discrete-time systems. IEEE Transactions on Circuits and Systems II: Express Briefs, 2014, vol. 61, no. 9, p. 716-720. DOI: 10.1109/TCSII.2014.2346688

[17] OPPENHEIM, A. V., ALAN, V. R., SCHAFER, W., et al. DiscreteTime Signal Processing. Prentice Hall, 1999. ISBN: 9788131704929

[18] ANTOUlas, A. C., SORENSEN, D. C., GUGERCIN, S. A Survey of Model Reduction Methods for Large-Scale Systems. [Online] 2000. Available at: https://hdl.handle.net/1911/101963

[19] CHAHLAOUI, Y. Benchmark Examples for Model Reduction. [Online] 2002. Available at: http://slicot.org/20-site/126-benchmarkexamples-for-model-reduction

\section{About the Authors...}

Sammana BATOOL is a PhD Scholar at Electrical Department, MCS, NUST. Her research interests include model order reduction.

Muhammad IMRAN is a PhD Scholar at Electrical Department, MCS, NUST. His research interests include model order reduction.

Dr. Muhammad IMRAN (corresponding author) is a faculty member at Electrical Department, MCS, NUST. His research interests include model order reduction.

Ehsan ELAHI is a MS student at Electrical Department, MCS, NUST. His research interests include model order reduction.

Ayesha MAQBOOL is a faculty member at Electrical Department, MCS, NUST. Her research interests include multiagent systems and machine learning.

Syed Amer Ahsan GILANI is a faculty member at Information Security Department, MCS, NUST. His research interests include double gimbal control moment gyroscopes for satellite attitude control. 